\title{
A moving lemma for algebraic cycles with modulus and contravariance
}

\author{
Wataru Kai*
}

\begin{abstract}
We prove a moving lemma which implies the contravariance of BlochEsnault's additive higher Chow group in smooth affine varieties and BindaSaito's higher Chow group (taken in the Nisnevich topology) in smooth varieties equipped with effective Cartier divisors. The new ingredients in the moving method are parallel translation with modulus in the affine space that involves a new integer parameter, and Noether's normalization lemma over a Dedekind base.
\end{abstract}

\section{Contents}

1 Preliminaries

2 Affine case

3 Linear projection method

4 Noether's normalization lemma over a Dedekind base

5 General case

6 Remarks on the simplicial version

\section{Introduction}

In recent years, the theory of algebraic cycles with modulus has been an attractive subject. It concerns the behavior of algebraic cycles at boundaries; more precisely, with how much multiplicity the cycle intersect a chosen effective Cartier divisor (called the modulus). The notion of modulus dates back at least to class field theory. In algebraic geometry, this concept has been studied since 1952 when Rosenlicht Ros52 introduced the divisor class group relative to a modulus (on a complete nonsingular curve).

*Mathematical Institute, Tohoku University, 6-3 Aramaki Aoba, Sendai 980-8578, Japan. kaiw@tohoku.ac.jp May 22, 2018 
The current development was initiated by Bloch and Esnault BE03 who introduced the additive higher Chow group $\operatorname{TCH}^{*}(X, n ; m)$ (a definitive version by Park (Par09]), and it has been a fruitful subject over the last decade. It is expected to have a relation to the relative $K$-groups

$$
K_{*}\left(X \times \mathbb{A}^{1}, X \times(m+1)\{0\}\right),
$$

just as Bloch's higher Chow group $\mathrm{CH}^{i}(X, n)$ is related to the $K$-groups:

$$
K_{n}(X)_{\mathbb{Q}} \cong \bigoplus_{i \geq 0} \mathrm{CH}^{i}(X, n)_{\mathbb{Q}}
$$

for smooth schemes $X$ over a field. One of the most remarkable results on this object [Rül07] is that for the spectrum of a field, the additive higher Chow groups in a "Milnor range" $\mathrm{TCH}^{n}(\operatorname{Spec}(k), n ; m)$ are isomorphic to the big de Rham-Witt $\mathbb{W}_{\{1, \ldots, m\}} \Omega_{k}^{n-1}$.

In the last couple of years, a more general theory of Chow groups with modulus had been anticipated: The Chow group of zero-cycles with modulus appeared in the work of Kerz and Saito [KS16] in relation to the class field theory with wild ramification of varieties over finite fields. Russell Rus13 defined a slightly different version earlier and studied its relation to his Albanese variety with modulus.

Binda and Saito BS17] then defined the higher Chow group with modulus $\mathrm{CH}^{i}(X \mid D, n)$ for an arbitrary pair of an algebraic scheme $X$ and an effective Cartier divisor $D$. It is defined as the homology groups of the cycle complex with modulus $z^{i}(X \mid D, \bullet)$. It contains all the groups above (in suitable versions) as particular cases. It is expected as a cycle-theoretic cohomology theory corresponding to the relative $K$-theory $K_{n}(X, D)$.

The complex $z^{i}(X \mid D, \bullet)$ is believed to produce a sensible cohomology theory at least if $X$ is smooth. For example, Binda-Saito [BS17, §4] have shown a zig-zag of quasi-isomorphisms $z^{1}(X \mid D, \bullet) \simeq \operatorname{ker}\left(\mathcal{O}^{*}(X) \rightarrow \mathcal{O}^{*}(D)\right)[1]$ when $X$ is local and factorial. Comparison maps between the Zariski or Nisnevich hypercohomology of $z^{i}(X \mid D, \bullet)$ and $K$-groups and de Rham(-Witt) cohomologies are known; latest results include [BS17] [RS15] [KP15] and [wa17.

In spite of being a candidate of a nice cohomology theory, it had been unknown if the additive higher Chow group and the higher Chow group with modulus are contravariant for arbitrary morphisms of smooth schemes. In the projective case, this was settled by Krishna and Park [KP12, KP17, but in the general case (e.g. affine) the concept of "modulus" gets harder to handle.

The aim of this paper is to provide an affirmative answer to this problem at least locally by proving a moving lemma. The moving lemma assures the contravariance of the additive higher Chow group in smooth affine schemes, and that of the Nisnevich hypercohomology of Binda-Saito's cycle complex in pairs $(X, D)$ for which $X \backslash D$ is smooth.

Let us explain our results in more detail: 


\section{Moving lemma}

We will often consider pairs $(X, D)$ consisting of an equi-dimensional scheme $X$ over a base field $k$ and an effective Cartier divisor $D$ on it.

For any integer $i \geq 0$, Binda and Saito defined a subcomplex $z^{i}(X \mid D, \bullet)$ of Bloch's cycle complex (cubical version); in particular, elements of $z^{i}(X \mid D, p)$ are represented by cycles on $X \times \mathbb{A}^{p}$ satisfying certain conditions. When $D=\emptyset$ it reduces to Bloch's higher Chow theory.

The complex is contravariant for flat maps. The association

$$
(U \stackrel{\text { étale }}{\longrightarrow} X) \mapsto z^{i}\left(U \mid D_{U}, \bullet\right)
$$

defines a presheaf on the small étale site on $X$. We will denote this presheaf by $z^{i}\left(-\mid D_{-}, \bullet\right)$.

Definitioin 1. For a finite collection $w$ of irreducible locally closed subsets of $X \backslash D$, define a subcomplex

$$
z_{w}^{i}(X \mid D, \bullet) \subset z^{i}(X \mid D, \bullet)
$$

by the condition that $V \in z^{i}(X \mid D, p)$ is in $z_{w}^{i}$ if and only if its support intersects $W \times F$ properly for every $W \in w$ and face $F$ of $\mathbb{A}^{n}$. This extends to a subcomplex of presheaves $z_{w}^{i}\left(-\mid D_{-}, \bullet\right) \subset z^{i}\left(-\mid D_{-}, \bullet\right)$.

Theorem 2 (Moving Lemma; see Theorem 1.6). Let $X$ be a scheme of finite type over a field, $D$ an effective Cartier divisor on $X$ and $w$ a finite set of irreducible locally closed subsets of $X \backslash D$. Assume $X \backslash D$ is smooth. Then the above inclusion is a quasi-isomorphism in the Nisnevich topology on $X$ :

$$
z_{w}^{i}\left(-\mid D_{-}, \bullet\right) \underset{\text { Nis }}{\hookrightarrow} z^{i}\left(-\mid D_{-}, \bullet\right)
$$

Actually, we prove it in a more general form (Theorem 1.6) which we need in a related work IK16] with Ryomei Iwasa.

\section{Noether's normalization lemma over a Dedekind base}

Along the proof of Theorem 2, we prove the following version of Noether's normalization lemma which can be of an independent interest. It is an analog of [Lev06, Th.10.2.2] and the same method has been applied in [SS18] to prove a version of Gabber's presentation theorem over a Dedekind base.

Theorem 3 (Theorem 4.1). Let $X \rightarrow B$ be an equi-dimensional morphism of relative dimension $n$, with $B$ the spectrum of a Dedekind domain. Then locally in the Nisnevich topology on $X$ and $B$, there is a finite surjective map

$$
X \rightarrow \mathbb{A}_{B}^{n}
$$

This explains the need of the Nisnevich topology in Theorem 2 from the technical side. 


\section{Functoriality of motivic cohomology}

As a direct consequence of Theorem 2, one gets the following functoriality. We explain how it can be deduced in $₫ 1$.

Theorem 4. Let $\left(X^{\prime}, D^{\prime}\right),(X, D)$ be pairs of equi-dimensional schemes and effective Cartier divisors, and assume $X \backslash D$ is smooth. Let $f: X^{\prime} \rightarrow X$ be a morphism which induces a morphism $D^{\prime} \rightarrow D$ of subschemes. Then there is a natural map

$$
f^{*}: z^{i}\left(-\mid D_{-}, \bullet\right) \rightarrow f_{*}\left(z^{i}\left(-\mid D_{-}^{\prime}, \bullet\right)\right)
$$

in the Nisnevich local derived category of abelian presheaves on $X$. Consequently there are natural pull-back maps on the hypercohomology groups

$$
f^{*}: \mathbb{H}^{n}\left(X_{\mathrm{Nis}}, z^{i}\left(-\mid D_{-}, \bullet\right)\right) \rightarrow \mathbb{H}^{n}\left(X_{\mathrm{Nis}}^{\prime}, z^{i}\left(-\mid D_{-}^{\prime}, \bullet\right)\right) .
$$

Unfortunately, it is known that the Nisnevich hypercohomology groups are not the same as the naive homology groups of the cycle complex in general; see e.g. [BS17, §2.1.4]. We think that the Nisnevich hypercohomology groups are a better invariant, especially because the $K$-theory satisfies the Nisnevich descent. It would be an interesting problem to ask if the Zariski hypercohomology coincides with the Nisnevich. We also mention that one gets a product structure in the same derived category, where $D$ and $E$ are two divisors on $X$ :

$$
z^{i}\left(-\mid D_{-}, \bullet\right) \otimes z^{j}\left(-\mid E_{-}, \bullet\right) \rightarrow z^{i+j}\left(-\mid(D+E)_{-}, \bullet\right) .
$$

A "projective" variant of the functoriality, without the need of Nisnevich localization, was proved by Krishna and Park [KP17].

\section{Additive higher Chow groups}

Additive higher Chow groups are obtained as a special case of higher Chow groups with modulus:

Definitioin 5. For a scheme $X$ and modulus $m \geq 1$, define

$$
T z^{i}(X, \bullet ; m):=z^{i}\left(X \times \mathbb{A}^{1} \mid X \times(m+1)\{0\}, \bullet-1\right)
$$

and let us call it the additive cycle complex (there are several notation/indexing conventions). Its homology groups

$$
\operatorname{TCH}^{i}(X, n ; m):=H_{n}\left(T z^{i}(X, \bullet ; m)\right)
$$

are called additive higher Chow groups of $X$.

The adjective "additive" was given because its values are often something additive, such as extensions of $k$-vector spaces as in the result of Rülling mentioned above.

One can prove analogs of the above results for this situation, and in particular, gets the functoriality of the additive higher Chow group in smooth affine schemes (without Nisnevich topology): See Theorem 1.7 
In the main body of the text, we will stick to the case of higher Chow groups with modulus, and make necessary comments for the additive case as needed.

The projective case had been settled by Krishna and Park [KP12].

\section{Organization of the paper}

In $\$ 1$, we recall the definition of the cycle complex and define certain subcomplexes of it. Along the way, we explain how the functoriality (Theorem 4) follows from the Moving Lemma 2

Sections $\S \$ 2[5$ are devoted to the proof of Moving Lemma 2 Of course, the whole proof follows the strategy exploited for Bloch's higher Chow theory Lev98, Part I, Chap. II, §3.5], which originates from that devised by Chow Cho56.

In $₫ 2$, we treat a special case of Theorem 2 to which the general case will be reduced. Here we need a slightly new moving construction which could be called parallel translation with modulus.

In 93 , we recall the common method of linear projection used in the reduction process. There is nothing new here. I have tried to clarify some details with which I had difficulties when learning this technique through the literature.

In 4 , we prove Noether's normalization lemma over a Dedekind base (Theorem 3), another ingredient used in the reduction process.

Finally in $\$$, we complete the proof of the moving lemma.

In a supplementary section $\sqrt{6}$ we say a few words about the moving lemma for the simplicial variant of the cycle complex.

Acknowledgements This paper is based on the author's Ph.D. thesis. I thank my advisors Prof. Shuji Saito and Prof. Tomohide Terasoma for encouragements and guidance. Discussions with Hiroyasu Miyazaki helped me very much. Especially he introduced the literature Lev06 to me when I turned very pessimistic. I am grateful to Sawako for everyday support. During the work, I was supported by the Program for Leading Graduate Schools, MEXT, Japan, and by Japan Society for the Promotion of Science as a research fellow (JSPS KAKENHI Grant Number 15J02264). Lastly I thank my parents for, among thousands of other things, making it possible for me to pursue my study in mathematics.

\section{Preliminaries}

In this section, we recall Binda-Saito's cycle complex, and the notion of constructible subsets and functions on noetherian topological spaces. After that we define certain subcomplexes of the cycle complex determined by data of constructible subsets of the variety. 


\subsection{Cycle complex with modulus}

In this article, an algebraic scheme is a scheme of finite type over a base field. Algebraic cycles will always be supported on equi-dimensional algebraic schemes. This includes the case where a scheme is given over a discrete valuation ring and cycles are on its generic fiber.

By convention, the dimension of a topological space is a non-negative integer or $\infty$ if the space is non-empty, and we set $\operatorname{dim}(\emptyset):=-\infty$ so that the inequality $\operatorname{dim}(X) \leq n$ means $X=\emptyset$ whenever $n \leq-1$.

\section{1 .1}

We write $\square^{p}:=\operatorname{Spec}\left(\mathbb{Z}\left[t_{1}, \ldots, t_{p}\right]\right)$ (the $p$-dimensional cube). Consider the open immersion $\square^{p} \subset\left(\mathbb{P}^{1}\right)^{p}$ and denote by $F_{p}$ the effective Cartier divisor

$$
F_{p}:=\sum_{j=1}^{p}\left\{t_{j}=\infty\right\} \subset\left(\mathbb{P}^{1}\right)^{p} .
$$

When we work over a base ring, we often tacitly take their base change.

Let $X$ be a scheme equipped with an effective Cartier divisor $D$. A closed subset $V \subset(X \backslash D) \times \square^{p}$ is said to satisfy the modulus condition if the following is true: Let $\bar{V}^{N}$ be the normalization of the (reduced) closure $\bar{V}$ of $V$ in $X \times\left(\mathbb{P}^{1}\right)^{p}$. Then the inequality of Cartier divisors on $\bar{V}^{N}$ each of which is obtained as the pull-back:

$$
\left.D\right|_{\bar{V}^{N}} \leq\left. F_{p}\right|_{\bar{V}^{N}}
$$

holds.

\section{1 .2}

The faces of $\square^{p}$ are the closed subschemes defined by some of the equations $\left\{t_{j}=\varepsilon\right\}$ with $1 \leq j \leq p$ and $\varepsilon=0,1$. A closed subset $V \subset(X \backslash D) \times \square^{p}$ is said to satisfy the face condition if for every face $F$ of $\square^{p}$, the intersection $V \times \square^{p} F$ has codimension in $V$ at least $\operatorname{codim}_{\square^{p}}(F)$.

Definitioin 1.1 (Cycle complex with modulus BS17]). For a pair $(X, D)$ of an equi-dimensional algebraic scheme and an effective Cartier divisor on it, let $\underline{z}^{i}(X \mid D, p)$ be the group of codimension $i$ algebraic cycles on $(X \backslash D) \times \square^{p}$ whose supports satisfy the modulus and the face conditions.

The groups $\underline{z}^{i}(X \mid D, p)(p \in \mathbb{N})$ are known to form a cubical abelian group $\underline{z}^{i}(X \mid D, \bullet)$ (an observation of Krishna-Park [KP12]). In particular, we can consider the associated (naive) complex, also denoted by the same symbol, by giving the differentials $\sum_{j=1}^{p}(-1)^{j}\left(\partial_{j, 0}^{*}-\partial_{j, 1}^{*}\right)$. Here, $\partial_{j, \varepsilon}: \square^{p-1} \rightarrow \square^{p}$ is the immer-

sion of the face $\left\{t_{j}=\varepsilon\right\}:\left(t_{1}, \ldots, t_{p-1}\right) \mapsto\left(t_{1}, \ldots, \stackrel{j}{\varepsilon}, t_{j}, \ldots, t_{p-1}\right)$. Its associated non-degenerate complex $z^{i}(X \mid D, \bullet)$ is defined (in degree $p$ ) as the quotient of $\underline{z}^{i}(X \mid D, p)$ by the images of $\underline{z}^{i}(X \mid D, q)(q<p)$ by the degeneracy maps $\square^{p} \rightarrow \square^{q}$ 
(called the degenerate part). An alternative definition of $z^{i}(X \mid D, p)$ is as the subgroup $\bigcap_{j=1}^{p} \operatorname{ker}\left(\partial_{j, 0}^{*}\right)$. The two definitions are isomorphic by the canonical map. It also gives a splitting of $\underline{z}^{i}$ into $z^{i}$ and the degenerate part.

\subsection{Constructible subsets}

Let $X$ be a noetherian topological space. The set $\mathfrak{C}(X)$ of constructible subsets of $X$ is the smallest subset of the power set $\mathfrak{P}(X)$ of $X$ satisfying

- Open sets of $X$ are in $\mathfrak{C}(X)$;

- $\mathfrak{C}(X)$ is closed under finite union and intersection, and complement.

The pull-back of a constructible subset by a continuous map of noetherian spaces is again constructible. It is not hard to see that the constructible subsets are precisely the unions of finitely many locally closed subsets.

A dense constructible subset of an irreducible noetherian space contains a dense open subset DG67, 0 $0_{\text {III }} 9.2 .2$ ]. Note in particular that a dense constructible subset $Z$ of an algebraic scheme $X$ has the same dimension as the scheme, because $Z$ contains a dense open subset $U$ of $X$ and therefore $\operatorname{dim}(U) \leq$ $\operatorname{dim}(Z) \leq \operatorname{dim}(X)$.

\subsection{1}

When $T$ is a set, a function $h: X \rightarrow T$ is said to be constructible if its image is a finite set, and for every $t \in T$ the inverse image $h^{-1}(t)$ is a constructible subset of $X$. Note in this case that for any subset $S$ of $T$, its inverse image $h^{-1}(S)$ is a constructible subset of $X$.

Constructibility of a function $h$ is known to be equivalent to the following condition (DG67, 0 III 9.3.2]; we state it when $X$ is the underlying space of a scheme): For every $x \in X$, there is a non-empty open set of $\overline{\{x\}}$ where $h$ takes the constant value $h(x)$.

If two functions $h_{1}, h_{2}: X \rightrightarrows \mathbb{N}$ are constructible, then their maximum $x \mapsto$ $\max \left\{h_{1}(x), h_{2}(x)\right\}$ is again constructible.

Theorem 1.2 ([DG67, $\left.\left.\mathrm{IV}_{1} 1.8 .4\right]\right)$. For every morphism of finite type between noetherian schemes, the image of a constructible subset is again constructible.

Theorem 1.3 ([DG67, $\mathrm{IV}_{3}$ 13.1.3]). Let $f: X \rightarrow Y$ be a morphisms of finite type between noetherian schemes. Then the function

$$
\begin{aligned}
X & \rightarrow \mathbb{N} \\
x & \mapsto \operatorname{dim}_{x}\left(f^{-1}(f(x))\right)
\end{aligned}
$$

is upper semi-continuous; namely, for every interger $n$, the subset $\{x \in X \mid$ $\left.\operatorname{dim}_{s}\left(f^{-1}(f(x))\right) \geq n\right\}$ is closed in $X$. 
Here, the local dimension of a topological space $X$ at a point $x$ is the infimum of the dimensions of its neighborhoods $U$ in $X$ :

$$
\operatorname{dim}_{x}(X):=\inf _{x \in U \subset X} \operatorname{dim}(U) .
$$

For example, if $X$ is the union $S \cup C$ of two irreducible components which have dimensions 2 and 1 respectively, then the local dimension is 2 at the points of $S$ whereas it is 1 at the points of $C \backslash S$.

Corollary 1.4. Let $f: X \rightarrow Y$ be a morphism of finite type between noetherian schemes. Let $Z \subset X$ be a constructible subset. Then the function which to $y \in Y$ assigns $\operatorname{dim}\left(f^{-1}(y) \cap Z\right)$ is a constructible function on $Y$.

Proof. If $Z=X$, this is a direct consequence of the previous two theorems.

In general, write $Z$ as the union of finitely many locally closed subsets $Z=$ $\bigcup_{i} Z_{i}$, each of which has a scheme structure. Then we know $\operatorname{dim}\left(f^{-1}(y) \cap\right.$ $Z)=\max _{i}\left\{\operatorname{dim}\left(f^{-1}(y) \cap Z_{i}\right)\right\}$ because in an algebraic scheme (like $f^{-1}(y)$ ), the dimension of the union of finitely many constructible subsets equals the maximum of their dimensions. (The dimension is equal to the maximum of the transcendence degrees of the points involved, after all.)

Since each member of the right hand side is already shown to be constructible, our function is constructible.

\subsection{Subcomplex determined by constructible subsets}

The following is an essentially equivalent variant of the subcomplex denoted by the symbol $z_{\mathcal{W}, e}^{i}$ e.g. in [KP12, Lev98. I'd like to try the following new convention partly because this makes it easier to write down the functoriality conditions, and partly because the use of constructible subsets eliminates the choices of decompositions of constructible subsets into locally closed subsets.

Definitioin 1.5. (1) By an increasing family $\mathcal{C}=\left\{C_{d}\right\}_{d \in \mathbb{Z}}$ of constructible subsets of an equi-dimensional algebraic scheme $X$ we mean a sequence

$$
\cdots \subset C_{0} \subset C_{1} \subset C_{2} \subset \cdots
$$

of constructible subsets where $\operatorname{dim}\left(C_{d}\right) \leq d$ and $C_{d}=X$ for $d \geq \operatorname{dim}(X)$. In particular, when $d$ is negative, then $C_{d}$ is the empty set. We shall drop the adjective increasing because we are only interested in this type of families. The family $\mathcal{C}_{\text {triv }}$ on $X$ defined by $\emptyset=C_{0}=\cdots=C_{\operatorname{dim}(X)-1} \subsetneq C_{\operatorname{dim}(X)}=X$ will be called the trivial family.

(2) When $D \subset X$ is an effective Cartier divisor and $\mathcal{C}=\left\{C_{d}\right\}_{d \in \mathbb{Z}}$ is a family of constructible subsets of $X \backslash D$, we define the cubical subgroup

$$
\underline{z}_{\mathcal{C}}^{i}(\bullet):=\underline{z}_{\mathcal{C}}^{i}(X \mid D, \bullet)
$$

of $\underline{z}^{i}(\bullet):=\underline{z}^{i}(X \mid D, \bullet)$ by the condition: A cycle $V \in \underline{z}^{i}(p)$ is in $\underline{z}_{\mathcal{C}}^{i}(p)$ if and only if for every face $F$ of $\square^{p}$ and $d \in \mathbb{Z}$, the following inequality of dimensions holds:

$$
\operatorname{dim}\left(\left(C_{d} \times F\right) \cap|V|\right) \leq d+\operatorname{dim}(F)-i
$$


Note that for the trivial family $\mathcal{C}_{\text {triv }}$ on $X \backslash D$, one has $\underline{z}_{\mathcal{C}_{\text {triv }}^{i}}(\bullet)=\underline{z}^{i}(\bullet)$.

Given a finite set $w$ of irreducible locally closed subsets of $X \backslash D$ as in Introduction, we define the corresponding $\mathcal{C}$ by

$$
C_{d}:=\bigcup_{\substack{W \in w \\ \operatorname{dim}(W) \leq d}} W
$$

so that one recovers the complex $z_{w}^{i}(X \mid D, \bullet)$ in Introduction as $z_{\mathcal{C}}^{i}(X \mid D, \bullet)$.

Thus Theorem 2 can be stated in a slightly stronger from that the inclusion

$$
\underline{z}_{\mathcal{C}}^{i}\left(-\mid D_{-}, \bullet\right) \rightarrow \underline{z}^{i}\left(-\mid D_{-}, \bullet\right)
$$

be a quasi-isomorphism of presheaves on $X_{\mathrm{Nis}}$. Here we regard the left hand side by pulling back constructible subsets by the given étale maps $U \rightarrow X$.

See Theorem 1.6 below for the full statement that we prove in this paper.

\subsection{1}

To illustrate why the quasi-isomorphism (1) is useful, we would like to discuss some functorial behavior of families of constructible subsets. Let $f: X^{\prime} \rightarrow X$ be a map of algebraic schemes and $\mathcal{C}^{\prime}$ be a family of constructible subsets of $X^{\prime}$. We define its push-forward $f_{*} \mathcal{C}^{\prime}$ by

$$
\left(f_{*} \mathcal{C}^{\prime}\right)_{d}:=\bigcup_{e \in \mathbb{Z}}\left\{x \in X \mid \operatorname{dim}\left(C_{d+e}^{\prime} \times_{X} x\right) \geq e\right\} \quad \text { for } d<\operatorname{dim}(X)
$$

and $\left(f_{*} \mathcal{C}^{\prime}\right)_{d}:=X$ for $d \geq \operatorname{dim}(X)$. Of course, the union over $0 \leq e \leq \operatorname{dim}\left(X^{\prime}\right)$ gives the same subset, and hence it is a constructible subset of $X$ by Proposition 1.4 (Chevalley's theorem). We obviously have $\left(f_{*} \mathcal{C}^{\prime}\right)_{d} \subset\left(f_{*} \mathcal{C}^{\prime}\right)_{d+1}$ (because $\left.C_{d+e}^{\prime} \subset C_{d+1+e}^{\prime}\right)$ and $\operatorname{dim}\left(\left(f_{*} \mathcal{C}^{\prime}\right)_{d}\right) \leq d$ because if we write $C_{d}^{(e)}$ for the $e$-th summand in the right hand side of the formula, then one should have

$$
\begin{aligned}
\operatorname{dim}\left(C_{d}^{(e)}\right)+e & \leq \operatorname{dim}\left(C_{d+e}^{\prime}\right) \\
& \leq d+e
\end{aligned}
$$

When $X$ is also given a family $\mathcal{C}=\left\{C_{d}\right\}_{d \in \mathbb{Z}}$ of constructible subsets, we say the morphism $f$ is compatible with $\mathcal{C}^{\prime}$ and $\mathcal{C}$ if the relation $\left(f_{*} \mathcal{C}^{\prime}\right)_{d} \subset C_{d}$ holds for all $d \in \mathbb{Z}$.

\section{3 .2}

Let $\left(X^{\prime}, D^{\prime}\right)$ and $(X, D)$ be pairs of algebraic schemes and effective Cartier divisors together with families $\mathcal{C}^{\prime}$ and $\mathcal{C}$ of constructible subsets on $X^{\prime} \backslash D^{\prime}$ and $X \backslash D$ respectively.

Given a morphism of pairs $f:\left(X^{\prime}, D^{\prime}\right) \rightarrow(X, D)$, i.e., a morphism $X^{\prime} \rightarrow X$ which induces a morphism on the subschemes $D^{\prime} \rightarrow D$, we may consider the 
push-forward $f_{*} \mathcal{C}^{\prime}$ on $X \backslash D$ by seeing the members of $\mathcal{C}^{\prime}$ as constructible subsets of $X^{\prime} \backslash f^{-1}(D)$. Consequently, we may ask if $f$ is compatible with the families $\mathcal{C}^{\prime}$ and $\mathcal{C}$. Suppose it is. Then if moreover $f$ is of finite Tor dimension (which is a standard assumption when one wants to pull-back algebraic cycles), we have a well-defined pull-back map $f^{*}: \underline{z}_{\mathcal{C}}^{i}(X \mid D, \bullet) \rightarrow \underline{z}_{\mathcal{C}^{\prime}}^{i}(X \mid D, \bullet)$ as follows.

By the compatibility condition $\left(f_{*} \mathcal{C}^{\prime}\right)_{d} \subset C_{d}$, for every $V \in \underline{z}_{\mathcal{C}}^{i}(X \mid D, p)$ we have:

$$
\begin{aligned}
\operatorname{dim}\left(\left(C_{d}^{\prime} \times F\right) \cap f^{-1}(|V|)\right) & \leq \max _{e \in \mathbb{Z}}\left\{e+\operatorname{dim}\left(\left(C_{d-e} \times F\right) \cap|V|\right)\right\} \\
& \leq \max _{e \in \mathbb{Z}}\{e+(d-e+\operatorname{dim}(F))-i\} \\
& =d+\operatorname{dim}(F)-i
\end{aligned}
$$

so that we can define a cycle $f^{*} V$ on $X^{\prime} \times \square^{p}$ by Serre's Tor formula Ser75, Chap.V.C)] and it satisfies the necessary dimension conditions for being an element of $\underline{z}_{\mathcal{C}^{\prime}}^{i}\left(X^{\prime} \mid D^{\prime}, p\right)$. Its support satisfies the modulus condition as well because $|V|$ does (cf. similarly to the "containment lemma" [KP12, Prop.2.4]). Therefore the pull-back $f^{*} V$ is in $\underline{z}_{\mathcal{C}^{\prime}}^{i}\left(X^{\prime} \mid D^{\prime}, p\right)$.

In particular, by setting $\mathcal{C}^{\prime}$ to be the trivial family and $\mathcal{C}:=f_{*} \mathcal{C}_{\text {triv }}^{\prime}$, we get a diagram of inclusion and pull-back

$$
\underline{z}^{i}(X \mid D, \bullet) \supset \underline{z}_{\mathcal{C}}^{i}(X \mid D, \bullet) \stackrel{f^{*}}{\longrightarrow} \underline{z}^{i}\left(X^{\prime} \mid D^{\prime}, \bullet\right) .
$$

The quasi-isomorphism (11) allows us to regard this diagram as a defining datum of a morphism $\underline{z}^{i}\left(-\mid D_{-}, \bullet\right) \rightarrow f_{*}\left(\underline{z}^{i}\left(-\mid D_{-}^{\prime}, \bullet\right)\right)$ in the derived category $D\left(X_{\mathrm{Nis}}\right)$.

\subsection{3}

As an aside, the association $((X, D), \mathcal{C}) \mapsto \underline{z}_{\mathcal{C}}^{i}(X \mid D, \bullet)$ is naturally a presheaf on the category of pairs $(X, D)$ whose open part $X \backslash D$ is regular and equipped with a family $\mathcal{C}$ of constructible subsets with the notion of compatible morphisms above. We have the forgetful functor $((X, D), \mathcal{C}) \mapsto(X, D)$ to the category of such pairs, but usually there is no functor in the opposite direction unless we restrict to a subcategory of pairs where every morphism is equi-dimensional or to special types of diagrams (e.g. finite) in the category of pairs. Given the quasi-isomorphism (11), it is possible to construct a presheaf on the category of pairs $(X, D)$ with $X \backslash D$ smooth which is objectwise quasi-isomorphic to our cycle complex Nisnevich locally, by a categorical construction Lev08 involving huge homotopy limit and colimit.

\section{3 .4}

Next, let $f: X^{\prime} \rightarrow X$ be an equi-dimensional morphism of relative dimension $\operatorname{dim}\left(X^{\prime} / X\right)$ inducing a morphism of effective Cartier divisors $D^{\prime} \rightarrow D$, and 
suppose that $X$ is given a family $\mathcal{C}$ of constructible subsets. Then we can define its pull-back $f^{*} \mathcal{C}$ by

$$
\left(f^{*} \mathcal{C}\right)_{d}:=f^{-1}\left(C_{d-\operatorname{dim}(X / Y)}\right) \backslash f^{-1}(D) .
$$

If moreover $f$ is of finite Tor dimension over $X \backslash D$ (e.g. if $f$ is flat or $X \backslash D$ is regular), then we have a natural pull-back map

$$
\underline{z}_{\mathcal{C}}^{i}(X \mid D, \bullet) \rightarrow \underline{z}_{f^{*} \mathcal{C}}^{i}\left(X^{\prime} \mid D^{\prime}, \bullet\right)
$$

In particular, for a pair $(X, D)$ equipped with a family $\mathcal{C}$, we can consider the presheaf

$$
\underline{z}_{\mathcal{C}}^{i}\left(-\mid D_{-}, \bullet\right): \quad(U \stackrel{\phi}{\rightarrow} X) \mapsto \underline{z}_{\phi^{*} \mathcal{C}}^{i}\left(U \mid \phi^{*} D, \bullet\right)
$$

on $X_{\mathrm{Nis}}$. When $\mathcal{C}$ is the trivial family $\mathcal{C}_{\text {triv }}$, we will drop it from the notation.

We extend the definition slightly. Given an equi-dimensional scheme $\psi: Y \rightarrow$ $\operatorname{Spec}(k)$, we consider the presheaf

$$
\underline{z}_{\mathcal{C}}^{i}\left(-\times Y \mid D_{-} \times Y, \bullet\right): \quad(U \stackrel{\phi}{\rightarrow} X) \mapsto \underline{z}_{(\phi \times \psi) * \mathcal{C}}^{i}\left(U \times Y \mid \phi^{*} D \times Y, \bullet\right)
$$

on $X_{\text {Nis }}$. Let us denote the value also by $\underline{z}_{\mathcal{C}}^{i}\left(U \times Y \mid \phi^{*} D \times Y, \bullet\right)$ omitting the pull-back notation for $\mathcal{C}$, when it is convenient.

Allowing the extra $Y$ turns out useful in our related work [K16] with Ryomei Iwasa where $Y$ will be the projective space $\mathbb{P}^{r}$.

\subsection{5}

The following is our main result in the full strength.

Theorem 1.6. Let $(X, D)$ be a pair of an equi-dimensional $k$-scheme over a field $k$ and an effective Cartier divisor, and $\mathcal{C}$ an increasing family (Definition 1.5.) of constructible subsets of $X \backslash D$. Assume $X \backslash D$ is smooth. Let $Y$ be an equi-dimensional $k$-scheme. Then the inclusion map of the presheaves on $X_{\mathrm{Nis}}$ :

$$
\underline{z}_{\mathcal{C}}^{i}\left(-\times Y \mid D_{-} \times Y, \bullet\right) \rightarrow \underline{z}^{i}\left(-\times Y \mid D_{-} \times Y, \bullet\right)
$$

is a quasi-isomorphism in the Nisnevich topology.

Now we make some remarks before ending the section.

First, Theorem 1.6 for a finite field $k$ follows from the case of infinite fields by the usual trace (norm) argument using extensions of $k$ having coprime degrees. We have to be careful in this case because we are considering the stalks of presheaves. But it turns out that this does not pose a problem in view of Hensel's lemma saying that a Nisnevich neighborhood of a point of $X_{k^{\prime}}$ (where $k \subset k^{\prime}$ is a finite separable extension) can be refined by a Nisnevich neighborhood defined over $X$ up to the passage to connected components.

Second, Theorem 1.6 implies the same assertion for the non-degenerate complexes $z^{i}$ because they are direct summands of the naive complexes $\underline{z}^{i}$ in a 
functorial way. Of course we are primarily interested in the non-degenerate complexes. Because of this, and to simplify the notation, in the text below we state results with underlines $\underline{z}^{i}$ but often omit them in the arguments.

Lastly, the following variant of Theorem 1.6 implies the contravariant functoriality of additive higher Chow groups of smooth affine varieties. Their proofs are parallel, so we will mainly discuss the proof of Theorem 1.6 in what follows and indicate necessary adaptation of the argument along the way as we need.

Theorem 1.7. Let $D \subset \mathbb{A}_{k}^{n}$ be an effective Cartier divisor and $X$ a smooth affine $k$-scheme. Suppose a family $\mathcal{C}$ of constructible subsets of $X$ is given.

Then the inclusion

$$
\underline{z}_{\mathcal{C} \times \mathbb{A}^{n}}\left(X \times \mathbb{A}^{n} \mid X \times D, \bullet\right) \hookrightarrow \underline{z}^{i}\left(X \times \mathbb{A}^{n} \mid X \times D, \bullet\right)
$$

is a quasi-isomorphism of complexes of abelian groups.

Consequently, additive higher Chow groups are contravariant in smooth affine varieties.

Here, $\mathcal{C} \times \mathbb{A}^{n}$ is the family $\left\{C_{d-n} \times \mathbb{A}^{n}\right\}_{d \in \mathbb{Z}}$ of constructible subsets of $X \times \mathbb{A}^{n}$.

\section{Affine case}

In this section, we prove the following special case of Theorem 1.6.

Theorem 2.1. Let $K$ be a discrete valuation field with valuation ring $\mathcal{O}_{K}$, and $\pi \in \mathcal{O}_{K}$ any element in the maximal ideal. Then for any sequence $\mathcal{C}$ of constructible subsets of $\mathbb{A}_{\mathcal{O}_{K}}^{n}$ and any scheme $Y$ equi-dimensional over $\mathcal{O}_{K}$, the inclusion of complexes of abelian groups

$$
\underline{z}_{\mathcal{C}}^{i}\left(\mathbb{A}_{\mathcal{O}_{K}}^{n} \times \mathcal{O}_{K} Y \mid V(\pi), \bullet\right) \subset \underline{z}^{i}\left(\mathbb{A}_{\mathcal{O}_{K}}^{n} \times_{\mathcal{O}_{K}} Y \mid V(\pi), \bullet\right)
$$

is a quasi-isomorphism. Here, we denote by $V(\pi)$ the Cartier divisor defined by $\pi$.

We also record the following slightly more global version. It is useful in the context of additive higher Chow groups.

Theorem 2.2. Let $k$ be a field and $\pi\left(x_{1}, \ldots, x_{n}\right) \in k\left[x_{1}, \ldots, x_{n}\right]$ be a non-zero polynomial, which defines an effective Cartier divisor $D:=\{\pi(\boldsymbol{x})=0\}$ on $\mathbb{A}_{k}^{n}$. Suppose a family $\mathcal{C}$ of constructible subsets of $\mathbb{A}_{k}^{n} \backslash D$ and an equi-dimensional $k$-scheme $Y$ are given.

Then the inclusion of complexes of abelian groups

$$
\underline{z}_{\mathcal{C}}^{i}\left(\mathbb{A}_{k}^{n} \times Y \mid D \times Y, \bullet\right) \subset \underline{z}^{i}\left(\mathbb{A}_{k}^{n} \times Y \mid D \times Y, \bullet\right)
$$

is a quasi-isomorphism for every $i$.

In the proof of Theorem 1.6 below, we will indicate some modifications needed for Theorem 2.1. 
The homotopy operator We shall use the following homotopy operator. Let $\boldsymbol{v} \in \mathbb{A}^{n}\left(\mathcal{O}_{K}\right)$ be vector and $s \geq 0$ be an integer. Consider the map of $\mathcal{O}_{K}$-schemes

$$
\begin{aligned}
\Phi_{\boldsymbol{v}, s}: \quad \mathbb{A}_{\mathcal{O}_{K}}^{n} \times \mathbb{A}^{1} & \rightarrow \mathbb{A}_{\mathcal{O}_{K}}^{n} \\
(\boldsymbol{x}, t) & \mapsto \boldsymbol{x}+\pi^{s} t \boldsymbol{v} .
\end{aligned}
$$

When $t=0$, the map $\Phi_{\boldsymbol{v}, s}(-, 0): \mathbb{A}_{\mathcal{O}_{K}}^{n} \rightarrow \mathbb{A}_{\mathcal{O}_{K}}^{n}$ is the identity map and when $t=1$, the map $\Phi_{\boldsymbol{v}, s}(-, 1)$ is the translation by the vector $\pi^{s} \boldsymbol{v}$.

For every $p \geq 0$, let us identify $\mathbb{A}^{1} \times \square^{p}$ and $\square^{p+1}$ by identifying $\mathbb{A}^{1}$ with the last factor of $\square^{p+1}$. Then we can ask if the map

$$
\Phi_{\boldsymbol{v}, s} \times \operatorname{id}_{Y} \times \operatorname{id}_{\square^{p}}: \mathbb{A}_{\mathcal{O}_{K}}^{n} \times \mathbb{A}^{1} \times Y \times \square^{p} \rightarrow \mathbb{A}_{\mathcal{O}_{K}}^{n} \times Y \times \square^{p}
$$

pulls back a given element of $\underline{z}^{i}\left(\mathbb{A}_{\mathcal{O}_{K}}^{n} \times Y \mid V(\pi), p\right)$ into $\underline{z}_{\mathcal{C}}^{i}\left(\mathbb{A}_{\mathcal{O}_{K}}^{n} \times Y \mid V(\pi), p+\right.$ $1)$. Since the face condition is easily seen to be always satisfied, the problem is the modulus condition and the proper intersection with the sets $C_{d}$. We are going to show that it can be made true by choosing suitable $s$ and $\boldsymbol{v}$.

[For Theorem 2.2, we choose $\boldsymbol{v}$ as an element of $\mathbb{A}^{n}(k)$ and define the map $\Phi_{\boldsymbol{v}, s}: \mathbb{A}_{k}^{n} \times \mathbb{A}^{1} \rightarrow \mathbb{A}_{k}^{n}$ by the same formula $(\boldsymbol{x}, t) \mapsto \boldsymbol{x}+t \pi(\boldsymbol{x})^{s} \boldsymbol{v}$ and consider pull-back by $\Phi_{\boldsymbol{v}, s} \times \operatorname{id}_{Y} \times \operatorname{id}_{\square^{p}}: \mathbb{A}_{k}^{n} \times \mathbb{A}^{1} \times Y \times \square^{p} \rightarrow \mathbb{A}_{k}^{n} \times Y \times \square^{p}$.]

We keep the notation throughout this section. We omit the underline in $\underline{z}^{i}$ below to simplify the notation.

\section{$2.1 \quad$ Verifying the modulus condition}

Here we prove the following.

Proposition 2.3. Let $V \subset \mathbb{A}_{\mathcal{O}_{K}}^{n} \times Y \times \square^{p}$ be an irreducible closed subset satisfying the modulus condition with respect to the divisor $V(\pi)$. Then there is an integer $s(V) \geq 0$ such that the closed set

$$
\Phi_{\boldsymbol{v}, s}^{-1}(V) \subset \mathbb{A}_{\mathcal{O}_{K}}^{n} \times Y \times \square^{p+1}
$$

satisfies the modulus condition for every $s \geq s(V)$ and $\boldsymbol{v} \in \mathbb{A}^{n}\left(\mathcal{O}_{K}\right)$.

[This proposition is true for the case of Theorem 2.2 as it is.]

Since the problem is local on $Y$, we may assume $Y$ is affine. Write $\Phi:=\Phi_{\boldsymbol{v}, s}$ and let

$$
\Phi^{-1}(V)^{-} \subset \mathbb{A}_{\mathcal{O}_{K}}^{n} \times Y \times\left(\mathbb{P}^{1}\right)^{p+1}
$$

be the closure of $\Phi^{-1}(V)$. For each index $i \in\{1, \ldots, p+1\}$, denote by $\mathbb{P}_{i}^{1}$ the $i$-th factor of $\left(\mathbb{P}^{1}\right)^{p+1}$. For a subset $I \subset\{1, \ldots, p+1\}$, let us define

$$
U_{I}:=\prod_{i \in I}\left(\mathbb{P}_{i}^{1} \backslash\{0\}\right) \times \prod_{i^{\prime} \in I^{c}}\left(\mathbb{P}_{i^{\prime}}^{1} \backslash\{\infty\}\right)
$$

which is an open set of $\left(\mathbb{P}^{1}\right)^{p+1}$. On the set $U_{I}$, the functions $1 / t_{i}(i \in I)$ and $t_{i^{\prime}}\left(i^{\prime} \in I^{c}\right)$ are regular functions. The divisor $F_{p+1}$ is defined by $\prod_{i \in I} \frac{1}{t_{i}}$ in this region. 
We define $U_{I^{\prime}}^{\prime} \subset\left(\mathbb{P}^{1}\right)^{p}$ for a subset $I^{\prime} \subset\{1, \ldots, p\}$ in a similar way.

Since the modulus condition is a local condition on the closed subset in question, let us consider the modulus condition for

$$
\Phi^{-1}(V)_{I}^{-}:=\Phi^{-1}(V)^{-} \times\left(\mathbb{P}^{1}\right)^{p+1} U_{I}
$$

for subsets $I \subset\{1, \ldots, p+1\}$.

If $p+1 \notin I$, then the set $\Phi^{-1}(V)_{I}^{-}$satisfies the modulus condition for every $s \geq 0$ and $\boldsymbol{v}$ because it is the pull-back of $\bar{V} \times \times_{\left(\mathbb{P}^{1}\right)^{p}} U_{I}^{\prime}$ (which satisfies the modulus condition) by the map

$$
\Phi \times \operatorname{id}_{Y} \times \operatorname{id}_{\left(\mathbb{P}^{1}\right)^{p}}: \mathbb{A}_{\mathcal{O}_{K}}^{n} \times Y \times U_{I} \rightarrow \mathbb{A}_{\mathcal{O}_{K}}^{n} \times Y \times U_{I}^{\prime} .
$$

Now suppose $p+1 \in I$ and write $I^{\prime}:=I \backslash\{p+1\}$ which we often regard as a subset of $\{1, \ldots, p\}$. Let $\mathcal{I}$ be the ideal defining the integral closed subscheme

$$
\bar{V}_{I^{\prime}}:=\bar{V} \times_{\left(\mathbb{P}^{1}\right)^{p}} U_{I^{\prime}}^{\prime} \subset \mathbb{A}_{\mathcal{O}_{K}}^{n} \times Y \times U_{I^{\prime}}^{\prime}
$$

and let $\left\{f_{\lambda}\left(\boldsymbol{x}, \frac{1}{t_{i}}\left(i \in I^{\prime}\right), t_{i^{\prime}}\left(i^{\prime} \in I^{c}\right)\right)\right\}_{\lambda \in \Lambda}$ be a finite set that generates $\mathcal{I}$ (recall that we are assuming that $Y$ is affine).

Let us note the following trivial fact:

Lemma 2.4. Let $A$ be an integral domain and $\operatorname{Frac}(A)$ be its fraction field. Let $z, \pi \in A$ be two elements. Then the element $z / \pi$ of $\operatorname{Frac}(A)$ is integral over $A$ if and only if there is a homogeneous polynomial

$$
E(\alpha, \beta) \in A[\alpha, \beta]
$$

which is monic as an element of $(A[\beta])[\alpha]$ such that

$$
E(z, \pi)=0 \quad \text { in } A \text {. }
$$

(This is just the matter of clearing the denominators of an integral dependence relation $\left(\frac{z}{\pi}\right)^{d}+a_{1}\left(\frac{z}{\pi}\right)^{d-1}+\cdots+a_{d}=0$ in $\operatorname{Frac}(A), a_{j} \in A$.)

Now, since $V_{I}$ satisfies the modulus condition, Lemma 2.4 applied to $\bar{V}_{I}$ implies that we have a relation of the form

$$
E_{I}\left[\boldsymbol{x}, \frac{1}{t_{i}}, t_{i^{\prime}}\right]\left(\prod_{i \in I^{\prime}} \frac{1}{t_{i}}, \pi\right)=\sum_{\lambda \in \Lambda} b_{\lambda} f_{\lambda}
$$

where $E_{I}\left[\boldsymbol{x}, \frac{1}{t_{i}}, t_{i^{\prime}}\right](\alpha, \beta) \in \mathcal{O}\left(\mathbb{A}_{\mathcal{O}_{K}}^{n} \times Y \times U_{I^{\prime}}^{\prime}\right)[\alpha, \beta]$ is homogeneous in $\alpha, \beta$ and monic in $\alpha$, and $b_{\lambda} \in \mathcal{O}\left(\mathbb{A}_{\mathcal{O}_{K}}^{n} \times Y \times U_{I^{\prime}}^{\prime}\right)$.

Meanwhile, let us observe the following. Let $\operatorname{deg}_{\boldsymbol{x}}\left(f_{\lambda}\right)$ be the total degree of $f_{\lambda}$ with respect to the variables $\boldsymbol{x}$. Then as a general fact about closure, the regular functions

$$
\begin{aligned}
\phi_{\lambda}(\boldsymbol{x}, & \left.\frac{1}{t_{i}}\left(i \in I^{\prime}\right), t_{i^{\prime}\left(i^{\prime} \in I^{c}\right)}, \frac{1}{t}\right) \\
& :=f_{\lambda}\left(\boldsymbol{x}+\pi^{s} t \boldsymbol{v}, \frac{1}{t_{i}}, t_{i^{\prime}}\right) \cdot\left(\frac{1}{t}\right)^{\operatorname{deg}_{\boldsymbol{x}}\left(f_{\lambda}\right)}
\end{aligned}
$$


on $\mathbb{A}_{\mathcal{O}_{K}}^{n} \times Y \times U_{I}$ are in the defining ideal for $\Phi^{-1}(V)_{I}^{-}$because it is true on the locus where both $t$ and $\frac{1}{t}$ are regular.

Expanding the right hand side, it can be written as

$$
\begin{aligned}
\phi_{\lambda}\left(\boldsymbol{x}, \frac{1}{t_{i}}, t_{i^{\prime}}, \frac{1}{t}\right)= & f_{\lambda}\left(\boldsymbol{x}, \frac{1}{t_{i}}, t_{i^{\prime}}\right) \cdot\left(\frac{1}{t}\right)^{\operatorname{deg}_{\boldsymbol{x}}\left(f_{\lambda}\right)} \\
& +\pi^{s} g_{\lambda}\left(\boldsymbol{x}, \frac{1}{t_{i}}, t_{i^{\prime}}, \frac{1}{t}\right)
\end{aligned}
$$

for some $g_{\lambda} \in \mathcal{O}\left(\mathbb{A}_{\mathcal{O}_{K}}^{n} \times Y \times U_{I}\right)$.

Now, let $\operatorname{deg}\left(E_{I}\right)$ be the homogeneous degree of $E_{I}$ and set

$$
s(V):=\max \left(\left\{\operatorname{deg}\left(E_{I}\right)\right\}_{I} \cup\left\{\operatorname{deg}_{\boldsymbol{x}}\left(f_{\lambda}\right)\right\}_{I, \lambda}\right) .
$$

This depends only on $V$ and not on $s$ or $\boldsymbol{v}$. Suppose $s \geq s(V)$. Then we may multiply (3) by $\left(\frac{1}{t}\right)^{s-\operatorname{deg}_{\boldsymbol{x}}\left(f_{\lambda}\right)} b_{\lambda}\left(\boldsymbol{x}, \frac{1}{t_{i}}, t_{i^{\prime}}\right)$ and sum them up with respect to $\lambda \in \Lambda$; formula (21) then gives

$$
\begin{aligned}
E_{I} & \left(\prod_{i \in I^{\prime}} \frac{1}{t_{i}}, \pi\right) \cdot\left(\frac{1}{t}\right)^{s}+\pi^{s} g\left(\boldsymbol{x}, \frac{1}{t_{i}}, t_{i^{\prime}}, \frac{1}{t}\right) \\
& =\sum_{\lambda \in \Lambda} b_{\lambda}\left(\boldsymbol{x}, \frac{1}{t_{i}}, t_{i^{\prime}}\right) \cdot\left(\frac{1}{t}\right)^{s-\operatorname{deg}_{\boldsymbol{x}}\left(f_{\lambda}\right)} \cdot \varphi_{\lambda}\left(\boldsymbol{x}, \frac{1}{t_{i}}, t_{i^{\prime}}, \frac{1}{t}\right)
\end{aligned}
$$

(with $g\left(\boldsymbol{x}, \frac{1}{t_{i}}, t_{i^{\prime}}, \frac{1}{t}\right) \in \mathcal{O}\left(\mathbb{A}_{\mathcal{O}_{K}}^{n} \times Y \times U_{I}\right)$ ) whose right hand side is in the defining ideal for $\Phi^{-1}(V)_{I}^{-}$.

Write $E_{I}$ as the sum of its leading term and the other: $E_{I}(\alpha, \beta)=\alpha^{\operatorname{deg}\left(E_{I}\right)}+$ $E_{I}^{\prime}(\alpha, \beta)$. Then the left hand side of formula (4) multiplied by $\left(\prod_{i \in I^{\prime}} \frac{1}{t_{i}}\right)^{s-\operatorname{deg}\left(E_{I}\right)}$ reads

$$
\left(\frac{1}{t} \prod_{i \in I} \frac{1}{t_{i}}\right)^{s}+E_{I}^{\prime}\left(\prod_{i \in I} \frac{1}{t_{i}}, \pi\right) \cdot\left(\frac{1}{t}\right)^{s}\left(\prod_{i \in I^{\prime}} \frac{1}{t_{i}}\right)^{s-\operatorname{deg}\left(E_{I}\right)}+\pi^{s} h
$$

with $h \in \mathcal{O}\left(\mathbb{A}_{\mathcal{O}_{K}}^{n} \times Y \times U_{I}\right)$. Clearly, this can be written as $\tilde{E}_{I}\left(\prod_{i \in I} \frac{1}{t_{i}}, \pi\right)$ for an $\tilde{E}_{I}\left[\boldsymbol{x}, \frac{1}{t_{i}}, t_{i^{\prime}}, \frac{1}{t}\right](\alpha, \beta) \in \mathcal{O}\left(\mathbb{A}_{\mathcal{O}_{K}}^{n} \times Y \times U_{I}\right)[\alpha, \beta]$ which is homogeneous in $\alpha, \beta$ of degree $s$ and monic in $\alpha$. By Lemma 2.4 again (applied to irreducible components of $\left.\Phi^{-1}(V)_{I}^{-}\right)$, we conclude that the closed subset $\Phi^{-1}(V)_{I}^{-}$satisfies the modulus condition on this region. Since this is true for all $I$, Proposition 2.3 has been proven.

[As is clear from the proof, this choice of $s(V)$ works universally, in the sense that the conclusion of the lemma is true after any extension of DVRs $\mathcal{O}_{K} \subset \mathcal{O}^{\prime}$, though we do not need this.] 
Remark 1. There is a way of proving Theorem 2.1 without proving Proposition 2.3. It consists of pulling back a cycle by $\Phi_{\boldsymbol{v}, 1}$ with $\boldsymbol{v}$ sufficiently general (see the next subsection) and pushing it forward by the $d$-th power map $\square^{1} \rightarrow \square^{1}$ (in the last factor of $\square^{p+1}$ ) with high enough powers $d$; see [Miy16a, §4.2.3].

Nonetheless, I chose to preserve Proposition 2.3 partly because my method has not appeared in the literature and partly because the method has been applied in [KP15] for their "fs-moving lemma."

\section{$2.2 \quad$ Verifying proper intersection}

We keep our notation: Namely, let $\mathcal{O}_{K}$ be a discrete valuation ring and $\pi \in \mathcal{O}_{K}$ an element in the maximal ideal. Let $\mathcal{C}=\left\{C_{d}\right\}_{d}$ be a sequence of constructible subsets on $\mathbb{A}_{K}^{n}$ and $Y$ be a scheme equi-dimensional over $\mathcal{O}_{K}$, with fiber dimension $\operatorname{dim}\left(Y / \mathcal{O}_{K}\right)$. Let $s \geq 0$ be an integer and the symbol $\boldsymbol{v}$ denote elements of $\mathbb{A}^{n}\left(\mathcal{O}_{K}\right)$. We are interested in pull-back of cycles by the morphism

$$
\begin{aligned}
& \Phi_{\boldsymbol{v}, s} \times \operatorname{id}_{Y} \times \operatorname{id}_{\square^{p}}: \quad \mathbb{A}_{\mathcal{O}_{K}}^{n} \times_{\mathcal{O}_{K}} Y \times \square^{p} \times \mathbb{A}^{1} \rightarrow \mathbb{A}_{\mathcal{O}_{K}}^{n} \times_{\mathcal{O}_{K}} Y \times \square^{p} \\
& (\boldsymbol{x}, y, \boldsymbol{t}, t) \quad \mapsto \quad\left(\boldsymbol{x}+\pi^{s} t \boldsymbol{v}, y, \boldsymbol{t}\right) .
\end{aligned}
$$

It is useful to allow more general vectors $\boldsymbol{v}$ when we discuss properties of a general $\boldsymbol{v}$. Namely, for any ring homomorphism $\mathcal{O}_{K} \rightarrow A$ and a vector $\boldsymbol{v} \in$ $\mathbb{A}^{n}(A)$, this morphism makes sense as a morphism from $\mathbb{A}_{A}^{n} \times \mathcal{O}_{K} Y \times \square^{p} \times \mathbb{A}^{1}$. We are primarily interested in the case where $A$ is the fraction field $K$ or its extension.

When we say some condition is true for a general $\boldsymbol{v}$, we mean that there is a dense open subset $U \subset \mathbb{A}_{K}^{n}$ such that the condition is true for every vector of the form $v$ : $\operatorname{Spec}(\Omega) \rightarrow U$, with $\Omega$ a field. Here, we concern only the generic fiber $\mathbb{A}_{K}^{n}$ because the conditions we consider will depend only on what happens over the generic point of $\operatorname{Spec}\left(\mathcal{O}_{K}\right)$.

Proposition 2.5. Let $s \geq 0$ be an integer and $V \subset \underline{z}^{i}\left(\mathbb{A}_{\mathcal{O}_{K}}^{n} \times_{\mathcal{O}_{K}} Y \mid V(\pi), p\right)$ be a cycle. Then a general $\boldsymbol{v} \in \mathbb{A}_{K}^{n}$ satisfies the following: For every face $F$ of $\square^{p} \times \mathbb{A}^{1} \cong \square^{p+1}$ and integer $d \in \mathbb{Z}$, we have the inequality of dimension:

$$
\begin{aligned}
& \operatorname{dim}\left(\left[C_{d} \times_{\mathcal{O}_{K}} Y \times(F \backslash\{t=0\})\right] \cap\left|\Phi_{\boldsymbol{v}, s}^{*}(V)\right|\right) \\
& \quad \leq\left[\operatorname{dim}\left(C_{d}\right)+\operatorname{dim}\left(Y / \mathcal{O}_{K}\right)+\operatorname{dim}(F)\right]-i .
\end{aligned}
$$

Consequently, if this $\boldsymbol{v}$ comes from an element of $\mathbb{A}^{n}\left(\mathcal{O}_{K}\right)$, it follows that the cycle $\left.\Phi_{\boldsymbol{v}, s}^{*}(V)\right|_{t=1}$ is in the subgroup $\underline{z}_{\mathcal{C}}^{i}\left(\mathbb{A}_{\mathcal{O}_{K}}^{n} \times_{\mathcal{O}_{K}} Y \mid V(\pi), p\right)$; if moreover $V$ happens to be in the subgroup $\underline{z}_{\mathcal{C}}^{i}\left(\mathbb{A}_{\mathcal{O}_{K}}^{n} \times_{\mathcal{O}_{K}} Y \mid V(\pi), p\right)$, then $\Phi_{\boldsymbol{v}, s}^{*}(V)$ is in $\underline{z}_{\mathcal{C}}^{i}\left(\mathbb{A}_{\mathcal{O}_{K}}^{n} \times_{\mathcal{O}_{K}} Y \mid V(\pi), p+1\right)$.

The following proof is essentially the same as that for a criterion given in [Blo86, Lem.1.1] which is often cited in this area. Here it seems to be more reader-friendly to give a direct proof than to make an obscure coordinate change so that we can apply the criterion.

Since there are only finitely many faces in a cube and data of (non-trivial) subsets $C_{d}$, we fix a face $F$ of $\square^{p} \times \mathbb{A}^{1}$ and an integer $d$. Let $\Sigma$ be the subset 
of $\mathbb{A}_{K}^{n} \times \mathbb{A}_{K}^{n} \times\left(\mathbb{A}_{K}^{n} \times Y_{K} \times(F \backslash\{t=0\})\right)$ consisting of points $\left(\boldsymbol{v}, \boldsymbol{x}^{\prime},(\boldsymbol{x}, y, \boldsymbol{t}, t)\right)$ satisfying the following four conditions:

- $\boldsymbol{x}^{\prime} \in C_{d}$;

- $(\boldsymbol{x}, y, \boldsymbol{t}) \in|V|$;

- $\boldsymbol{x}=\boldsymbol{x}^{\prime}+\pi^{s} t \boldsymbol{v}$.

We want to consider $\Sigma$ because the set $\left[C_{d} \times_{\mathcal{O}_{K}} Y \times(F \backslash\{t=0\})\right] \cap\left|\Phi_{\boldsymbol{v}, s}^{*}(V)\right|$ can be recovered as follows: Look at the fiber $\Sigma_{\boldsymbol{v}}$ of the projection to the $\boldsymbol{v}$ factor $\Sigma \rightarrow \mathbb{A}_{K}^{n}$ above the chosen point $\boldsymbol{v}$ and take the image by the projection $\Sigma_{\boldsymbol{v}} \rightarrow \mathbb{A}_{K}^{n} \times Y_{K} \times(F \backslash\{t=0\})$ to the $\left(\boldsymbol{x}^{\prime}, y, \boldsymbol{t}, t\right)$-factors; then this is the set we are interested in.

Now, for every pair of $\boldsymbol{x}^{\prime} \in C_{d}$ and $(\boldsymbol{x}, y, \boldsymbol{t}, t) \in\left(|V| \times \mathbb{A}^{1}\right) \times_{\square^{p+1}}(F \backslash\{t=$ $0\}$ ), there is a unique $\boldsymbol{v} \in \mathbb{A}_{K}^{n}$ that makes the third condition true (namely $\left.\boldsymbol{v}:=\left(\boldsymbol{x}-\boldsymbol{x}^{\prime}\right) / \pi^{s} t\right)$. It follows that

$$
\begin{aligned}
\operatorname{dim}(\Sigma) & =\operatorname{dim}\left(C_{d}\right)+\operatorname{dim}\left(\left(|V| \times \mathbb{A}^{1}\right) \times_{\square^{p}+1}(F \backslash\{t=0\})\right) \\
& \leq \operatorname{dim}\left(C_{d}\right)+\operatorname{dim}\left(\mathbb{A}_{K}^{n} \times Y_{K} \times F\right)-i \quad \text { [the face condition] }
\end{aligned}
$$

Therefore, the projection to the $\boldsymbol{v}$-factor $\Sigma \rightarrow \mathbb{A}_{K}^{n}$ has generic fiber with dimension $\leq \operatorname{dim}\left(C_{d}\right)+\operatorname{dim}\left(Y_{K} \times F\right)-i$, cf. Proposition 1.4 (Chevalley's theorem). This complets the proof of Proposition 2.5.

[For Theorem 2.2, one needs the inequality

$$
\begin{aligned}
& \operatorname{dim}\left(\left[C_{d} \times Y \times(F \backslash\{t=0\})\right] \cap\left|\Phi_{\boldsymbol{v}, s}^{*}(V)\right|\right) \\
& \quad \leq\left[\operatorname{dim}\left(C_{d}\right)+\operatorname{dim}(Y)+\operatorname{dim}(F)\right]-i .
\end{aligned}
$$

The above proof works if one defines $\Sigma \subset \mathbb{A}_{k}^{n} \times \mathbb{A}_{k}^{n} \times\left(\mathbb{A}_{k}^{n} \times Y \times(F \backslash\{t=0\})\right)$ by the same formula.]

\subsection{Proof of the affine case}

Now we can prove that the inclusion

$$
\underline{z}_{\mathcal{C}}^{i}\left(\mathbb{A}_{\mathcal{O}_{K}}^{n} \times_{\mathcal{O}_{K}} Y \mid V(\pi), \bullet\right) \rightarrow \underline{z}^{i}\left(\mathbb{A}_{\mathcal{O}_{K}}^{n} \times_{\mathcal{O}_{K}} Y \mid V(\pi), \bullet\right)
$$

induces isomorphisms on the homology groups.

For the surjectivity of the map on the homology, choose any homology class in the target and a cycle $V \in \underline{z}^{i}\left(\mathbb{A}_{\mathcal{O}_{K}}^{n} \times_{\mathcal{O}_{K}} Y \mid V(\pi), p\right)$ representing it. Take a large enough $s \geq 0$ using Proposition 2.3 and then a vector $\boldsymbol{v} \in \mathbb{A}_{\mathcal{O}_{K}}^{n}$ whose generic fiber is sufficiently general using Proposition 2.5. We conclude that the cycle $\left.\Phi_{\boldsymbol{v}, s}^{*}(V)\right|_{t=1}$ is in the subcomplex $\underline{z}_{\mathcal{C}}^{i}\left(\mathbb{A}_{\mathcal{O}_{K}}^{n} \times_{\mathcal{O}_{K}} Y \mid V(\pi), p\right)$ and the obvious compatibility tells us

$$
\left.\Phi_{\boldsymbol{v}, s}^{*}(V)\right|_{t=1}-V=(-1)^{p} \partial\left(\Phi_{\boldsymbol{v}, s}^{*}(V)\right)
$$


where $\partial$ denotes the differential in the cycle complex. It follows that the map on the homology is surjective.

The proof of the injectivity is similar: We take any cycle $W$ representing a homology class in the source, suppose it becomes a boundary $W=\partial V$ in the target and choose $s \geq 0$ and $\boldsymbol{v}$ such that $\Phi_{\boldsymbol{v}, s}^{*}$ works for $V$. The point is that the inclusion in question is a weak homotopy equivalence, meaning that even if we might not have a uniform homotopy inverse, we do have a homotopy inverse on every finitely generated subcomplex.

This completes the proof of Theorem 2.1.

[The corresponding step for the proof of Theorem 2.2 is the same.]

\section{$3 \quad$ Linear projection method}

We collect some general facts on the technique of "moving by linear projections" $\mathbb{A}^{N} \rightarrow \mathbb{A}^{n}(N \geq n)$.

Let us write $\mathbb{A}^{N}=\operatorname{Spec}\left(\mathbb{Z}\left[x_{1}, \ldots, x_{N}\right]\right)$ and $\mathbb{A}^{n}=\operatorname{Spec}\left(\mathbb{Z}\left[y_{1}, \ldots, y_{n}\right]\right)$.

\subsection{Notation}

Suppose we are given $n$ linear polynomials $\phi_{1}, \ldots, \phi_{n}$ in $N$ variables (i.e. an $N \times n$ matrix) with coefficients in some ring. They define a map of schemes $\phi: \mathbb{A}^{N} \rightarrow \mathbb{A}^{n}$ over the ring, defined by $\boldsymbol{x}=\left(x_{1}, \ldots, x_{N}\right) \mapsto\left(\phi_{1}(\boldsymbol{x}), \ldots, \phi_{n}(\boldsymbol{x})\right)$. The surjective linear maps form a dense open subset $M_{N \times n}^{*}$ of the space of all $N \times n$ matrices $M_{N \times n}$.

The following projective terminology is often useful as well. Let $\mathbb{A}^{N} \subset \mathbb{P}^{N}$ be the usual open immersion. Let $X_{0}, \ldots, X_{N}$ be the homogeneous coordinates so that $x_{i}=X_{i} / X_{0}(i=1, \ldots, N)$. We may think of the polynomials $\phi_{i}\left(x_{1}, \ldots, x_{N}\right)$ as the dehomogenization of the polynomials $\phi_{i}\left(X_{1}, \ldots, X_{N}\right)$.

Define a morphism $\Phi$ by

$$
\begin{aligned}
\Phi: \mathbb{P}^{N} \backslash V\left(\left(X_{0}, \phi_{1}(\boldsymbol{X}), \ldots, \phi_{n}(\boldsymbol{X})\right)\right) & \rightarrow \mathbb{P}^{n} \\
{\left[X_{0}: \cdots: X_{N}\right] } & \mapsto\left[X_{0}: \phi_{1}(\boldsymbol{X}): \cdots: \phi_{n}(\boldsymbol{X})\right]
\end{aligned}
$$

which is affine. Let us write $V(\phi):=V\left(\left(X_{0}, \phi_{1}(\boldsymbol{X}), \ldots, \phi_{n}(\boldsymbol{X})\right)\right)$. It can be checked that the following diagram:

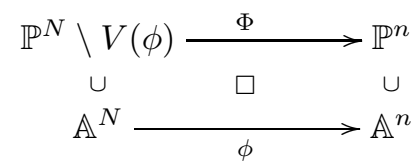

is cartesian.

If $\phi$ belongs to $M_{N \times n}^{*}$, the morphism $\Phi$ is an affine bundle with fiber $\mathbb{A}^{N-n}$. 


\subsection{Over a base field}

From now on, we work over a base field $k$, and $\phi$ will always be taken from $M_{N \times n}^{*}$.

When we say some property holds for a general $\phi \in M_{N \times n}^{*}$, it means that there is a dense open subset of $M_{N \times n}^{*}$ such that if a field-valued point $\operatorname{Spec}(\Omega) \rightarrow$ $M_{N \times n}^{*}$ maps into the subset, then the property holds for the corresponding map $\phi: \mathbb{A}_{\Omega}^{N} \rightarrow \mathbb{A}_{\Omega}^{n}$. Since the properties we consider are such that the points satisfying the property form a constructible subset of $M_{N \times n}^{*}$, this will always be equivalent to the condition that the property holds for the generic point of $M_{N \times n}^{*}$. Also, since $M_{N \times n}^{*}$ is of finite type over the field $k$, it is equivalent to its truth for the closed points of a dense open subset of $M_{N \times n}^{*}$. If $k$ is infinite, it can even be checked on $k$-rational points alone.

For a field extension $k \subset \Omega$, a matrix $\phi \in M_{N \times n}^{*}(\Omega)$ and a point $\mathbb{A}^{N}(\Omega)$, the fiber of $\phi: \mathbb{A}_{\Omega}^{N} \rightarrow \mathbb{A}_{\Omega}^{n}$ containing $x$ is $x+\operatorname{ker}(\phi)$.

Let $X \subset \mathbb{A}^{N}$ be a closed subset and $\bar{X}$ be its closure in $\mathbb{P}^{N}$. If $\bar{X}$ does not meet $V(\phi)$, then $\Phi_{\mid \bar{X}}$ is both affine and projective, hence finite (regardless of the scheme structure of $\bar{X})$. It follows that $\phi_{\mid X}$ is finite and $\operatorname{dim}(X) \leq n$ in this case. Conversely, if $X$ has dimension $\leq n$, this becomes the case for a general $\phi \in M_{N \times n}^{*}$.

\subsubsection{Avoiding a closed subset}

We keep the notation above.

Let $x \in \mathbb{A}^{N}$ be a point and $Z \subset \mathbb{A}^{N}$ be a closed subset of dimension $<n$ not containing $x$. Then for a general $\phi \in M_{N \times n}^{*}$, we have $\phi(x) \notin \phi(Z)$.

Proof. Define a closed subset $\Sigma \subset Z \times M_{N \times n}^{*}$ by

$$
\Sigma:=\{(z, \phi) \mid \phi(z)=\phi(x)\} .
$$

The fiber of a given $z \in Z$ along the projection $\Sigma \rightarrow Z$ is

$$
\left\{\phi \in M_{N \times n}^{*} \mid x \in z+\operatorname{ker}(\phi)\right\}
$$

(i.e. the vector $\vec{x} z$ connecting $x$ and $z$ is in the kernel of $\phi$ ). This set has codimension $n$ in $M_{N \times n}^{*}$ because $\overrightarrow{x z} \neq \mathbf{0}$ by the assumption $x \notin Z$. It follows that $\operatorname{dim}(\Sigma)=\operatorname{dim}\left(M_{N \times n}^{*}\right)+\operatorname{dim}(Z)-n<\operatorname{dim}\left(M_{N \times n}^{*}\right)$. Therefore the projection $\Sigma \rightarrow M_{N \times n}^{*}$ cannot be surjective. It follows that for the generic point $\phi \in M_{N \times n}^{*}$, there is no $z \in Z$ satisfying $\phi(z)=\phi(x)$.

\subsubsection{Smoothness}

We need to recall Veronese embeddings. Let $d \geq 1$ be an integer. Let $V \subset$ $k\left[x_{1}, \ldots, x_{N}\right]$ be the linear subspace of polynomials of degree $\leq d$. We will denote the affine space $\operatorname{Spec}(k[V])$ also by $V^{*}$. The Veronese embedding of degree $d$ is the closed embedding

$$
\mathbb{A}^{N} \hookrightarrow V^{*}
$$


corresponding to the surjective ring map $k[V] \rightarrow k\left[x_{1}, \ldots, x_{N}\right]$ induced by the inclusion of $V$ into the right hand side. Occasionally we consider the corresponding projective counterpart

$$
\mathbb{P}^{N} \hookrightarrow \mathbb{P}\left(k \oplus V^{*}\right) .
$$

The Veronese re-embedding of a given closed embedding $X \hookrightarrow \mathbb{A}^{N}$ refers to the composition with the $d$-fold Veronese embedding $X \rightarrow \mathbb{A}^{N} \hookrightarrow V^{*}$. When we say some property holds for a general $\phi \in M_{N \times n}^{*}$ after the Veronese re-embedding of some degree, we mean that it is true after composing $X \hookrightarrow \mathbb{A}^{N} \hookrightarrow V^{*}$ and writing $\mathbb{A}^{N}$ anew for $V^{*}$.

Let $X \subset \mathbb{A}^{N}$ be a closed subscheme of dimension $\geq n$ everywhere, and $x \in X$ be a point. Then the map $\phi_{\mid X}$ is smooth along $\phi^{-1} \phi(x) \cap X_{\mathrm{sm}}$ for a general $\phi \in M_{N \times n}^{*}$ after any Veronese re-embedding of degree $\geq 2$. (If $\operatorname{dim}(X)=n$, we do not need a Veronese re-embedding.)

This is a version of Bertini smoothness theorem; see [AGV71, XI, 2.1].

\subsubsection{Birationality}

Let $X \subset \mathbb{A}^{N}$ be a closed subscheme of dimension $\leq n$ and $x \in X_{\mathrm{sm}}$ be a point such that its closure has dimension $<n$. Then the extension of residue fields $k(\phi(x)) \rightarrow k(x)$ is trivial for a general $\phi \in M_{N \times n}^{*}$.

Proof. We prove the separability and the pure inseparability of the field extension separately.

First, for a general $\phi$, consider the composition of $\phi_{\mid X}$ and the projection to the first $\operatorname{dim}(X)$ factors:

$$
\bar{\phi}: X \stackrel{\phi_{\mid X}}{\longrightarrow} \mathbb{A}^{n} \rightarrow \mathbb{A}^{\operatorname{dim}(X)}
$$

which is étale at $x$ by 3.2 .2 . In particular, the extension $k(\bar{\phi}(x)) \rightarrow k(x)$ is finite and separable. Therefore, so is $k(\phi(x)) \rightarrow k(x)$.

Next, let $k \rightarrow k^{\text {perf }}$ be the perfect closure of $k$. Since it is a purely inseparable extension, there is a unique point $\tilde{x}$ on $\mathbb{A}_{k^{\text {perf }}}^{N}$ above $x$. Since $k^{\text {perf }}$ is a perfect field, the residue field $k^{\text {perf }}(\tilde{x})$ is separably generated over $k^{\text {perf }}$.

Put $Z:=\overline{\{x\}}$. Refreshing the notation, let $\bar{\phi}$ denote the composition

$$
\bar{\phi}: Z_{k^{\text {perf }}} \stackrel{\phi}{\rightarrow} \mathbb{A}_{k^{\text {perf }}}^{n} \rightarrow \mathbb{A}_{k^{\text {perf }}}^{\operatorname{dim}(Z)}
$$

where the second map is the projection to the first $\operatorname{dim}(Z)$ factors. By the first half of this proof, the extension $k^{\operatorname{perf}}(\bar{\phi}(\tilde{x})) \rightarrow k^{\text {perf }}(\tilde{x})$ finite and separable. Note that the assumption $\operatorname{dim}(Z)<n$ allows us to consider the function $y_{\operatorname{dim}(Z)+1}$. Its pull-back $\phi^{*}\left(y_{\operatorname{dim}(Z)+1}\right)$ has the form $c_{1} x_{1}+\cdots+c_{N} x_{N}$ with $c_{i} \in k^{\text {perf }}$ (or in some extension thereof). Since the functions $x_{1}, \ldots, x_{N}$ generate the 
field $k^{\text {perf }}(\tilde{x})$ over $k^{\text {perf }}(\bar{\phi}(\tilde{x}))$, by (the proof of) Primitive Element Theorem, a general $\phi \in M_{N \times n}^{*}$ makes the equality

$$
k^{\text {perf }}(\tilde{x})=k^{\operatorname{perf}}(\bar{\phi}(\tilde{x}))\left(\phi^{*}\left(y_{\operatorname{dim}(Z)+1}\right)\right)
$$

true. In particular one has $k^{\text {perf }}(\tilde{x})=k^{\text {perf }}(\phi(\tilde{x}))$

Now in the following diagram:

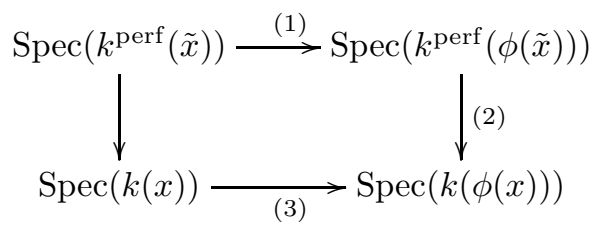

the arrow (1) is an isomorphism and (2) is purely inseparable. It follows that the arrow (3) is purely inseparable as well.

This completes the proof.

\subsubsection{Chow's moving lemma}

Let $X \subset \mathbb{A}^{N}$ be a closed subscheme of pure dimension $n$ and let $W \subset X$ be a constructible subset.

Suppose we are given an equi-dimensional scheme $Y$, an integer $n \geq 0$ and a closed subset $V$ of $X \times Y \times \square^{n}$.

For a map $\phi \in M_{N \times n}^{*}$ such that $\phi_{\mid X}$ is finite surjective, define a new closed subset $\phi^{+}(V)$ as the closure of $\left(\phi_{\mid X} \times \operatorname{id}_{Y \times \square^{n}}\right)^{-1}\left(\phi_{\mid X} \times \operatorname{id}_{Y \times \square^{n}}\right)(V) \backslash V$ in $X \times Y \times \square^{n}$.

Now we claim that the following inequality holds:

$$
\begin{array}{r}
\operatorname{dim}\left(\phi^{+}(V) \cap(W \times Y)\right) \leq \max \{\operatorname{dim}(V)+\operatorname{dim}(W)-\operatorname{dim}(X), \\
\operatorname{dim}(V \cap(W \times Y))-1\}
\end{array}
$$

for a general $\phi \in M_{N \times n}^{*}$, if $X$ is smooth at the images of the generic points of $V \cap(W \times Y)$.

Proof. Clearly, we may assume $n=0$ because $Y \times \square^{n}$ is equi-dimensional anyway.

Denote by $\operatorname{ram}\left(\phi_{\mid X}\right) \subset X$ the complement of the étale locus of $\phi_{\mid X}$.

Put $A:=V \cap(W \times Y) \cap \operatorname{ram}\left(\phi_{\mid X} \times \operatorname{id}_{Y}\right)$. Observe the obvious inclusion

$$
\phi^{+}(V) \cap(W \times Y) \quad \subset \quad A \cup\left[\phi^{+}(V) \cap(W \times Y) \backslash A\right]
$$

We are going to show

- $\operatorname{dim}(A) \leq \operatorname{dim}(V \cap(W \times Y))-1$ and

- $\operatorname{dim}\left(\phi^{+}(V) \cap(W \times Y) \backslash A\right) \leq \operatorname{dim}(V)+\operatorname{dim}(W)-\operatorname{dim}(X)$ 
for a general $\phi \in M_{N \times n}^{*}$.

First, since $X$ is assumed to be smooth at the images of the generic points of the set $V \cap(W \times Y)$, a general $\phi_{\mid X}$ is étale at these points. This shows the first inequality.

Next, consider the following constructible set:

$$
\begin{aligned}
& \ell(V, W):= \\
& \left\{(\ell, y) \mid \begin{array}{l}
\ell \text { is a line in } \mathbb{A}^{N}, y \in Y, \\
\exists\left(x_{1}, x_{2}\right) \in(X \times W) \backslash \Delta_{X} \text { such that } x_{1}, x_{2} \in \ell \text { and }\left(x_{1}, y\right) \in V
\end{array}\right\} .
\end{aligned}
$$

It is easy to see the inequality $\operatorname{dim}(\ell(V, W)) \leq \operatorname{dim}(V)+\operatorname{dim}(W)$. We have the "direction" map

$$
\delta: \ell(V, W) \rightarrow \mathbb{P}_{\infty}^{N-n} .
$$

Two points $x_{1}, x_{2} \in X$ are mapped by $\phi$ to the same point if and only if either $x_{1}=x_{2}$ or $x_{1} \neq x_{2}$ and $\delta\left(\overrightarrow{x_{1} x_{2}}\right) \in V(\phi)$.

Look at the following diagram of constructible sets:

$$
\begin{aligned}
& X \supset \phi^{+}(V) \cap(W \times Y) \backslash A \\
& \uparrow \mathrm{pr}_{1} \\
& \Sigma_{1}:=\{(x, \ell) \in W \times \ell(V, W) \mid x \in \ell \text { and } \delta(\ell) \in V(\phi)\} \\
& \downarrow \mathrm{pr}_{2} \\
& \delta^{-1}(V(\phi)) \subset \ell(V, W) \stackrel{\delta}{\rightarrow} \mathbb{P}_{\infty}^{N-1} .
\end{aligned}
$$

Now, since a general $\phi_{\mid X}$ is finite, the projection $\mathrm{pr}_{2}$ has finite fibers. It follows that $\operatorname{dim}\left(\Sigma_{1}\right)=\operatorname{dim}\left(\delta^{-1}(V(\phi))\right)$.

For each integer $j \geq 0$, write $S_{j}$ for the set of points of $\mathbb{P}_{\infty}^{N-1}$ at which the fiber of $\delta$ has dimension $\geq j$. Then if $V(\phi)$ meet the subsets $S_{j}$ properly in $\mathbb{P}_{\infty}^{N-1}$, the set $\delta^{-1}(V(\phi))$ has dimension $\ell(V, W)-n$ which is at most $\operatorname{dim}(V)+$ $\operatorname{dim}(W)-n$. This proper intersection is true for a general $\phi \in M_{N \times n}^{*}$ because it is about intersections of a linear subspace and subsets of $\mathbb{P}_{\infty}^{N-1}$. We obtain

$$
\operatorname{dim}\left(\Sigma_{1}\right) \leq \operatorname{dim}(V)+\operatorname{dim}(W)-\operatorname{dim}(X) .
$$

It remains to show that the image of $\operatorname{pr}_{1}$ contains $\phi^{+}(V) \cap(W \times Y) \backslash A$, because then we will obtain

$$
\operatorname{dim}\left(\phi^{+}(V) \cap(W \times Y) \backslash A\right) \leq \operatorname{dim}\left(\Sigma_{1}\right)
$$

which will finish the proof.

Let us observe that since $V$ is closed and $\phi_{\mid X}$ is finite (hence a closed map), the subset $\phi^{+}(V)$ is alternatively obtained by the following steps: consider the subset

$$
\Sigma_{2}=\left\{\left(z_{1}, z_{2}\right) \in((X \times Y) \backslash V) \times V \mid \phi\left(z_{1}\right)=\phi\left(z_{2}\right)\right\},
$$


take its closure $\overline{\Sigma_{2}}$ in $(X \times Y)^{2}$ and project it by the first projection $\overline{\Sigma_{2}} \hookrightarrow$ $(X \times Y)^{2} \rightarrow X \times Y$.

Lemma 3.1 (adaptation of [Rob71, Prop.4]). Keep the notation just above. Let $z=(x, y) \in X \times Y$ be a point. If the point $(z, z) \in(X \times Y)^{2}$ lies in $\overline{\Sigma_{2}}$, then there is a line $\ell \subset T_{X, x}$ with the property that $\phi(\ell) \in V(\phi)$.

Let us see how Lemma 3.1 implies the inclusion $\phi^{+}(V) \cap(W \times Y) \backslash A \subset$ image $\left(\operatorname{pr}_{1}\right)$. Suppose we are given a point $z_{1}=\left(x_{1}, y\right) \in W \times Y$ which is in $\phi^{+}(V)$. By its definition, there is a point $z_{2}=\left(x_{2}, y\right) \in V$ with $\phi\left(x_{1}\right)=\phi\left(x_{2}\right)$. If $z_{1} \neq z_{2}$, then $\left(z_{1}, \overrightarrow{z_{1}} \overrightarrow{z_{2}}\right)$ gives a point whose first projection is $z_{1}$. If $z_{1}=z_{2}$, then Lemma 3.1 implies that there is a line $\ell \subset T_{X, x_{1}}$ with $\delta(\ell) \in V(\phi)$, which implies that $\phi_{\mid X}$ is not étale at this point. It follows that the point $z_{1}=z_{2}$ is in $V \cap(W \times Y) \cap \operatorname{ram}\left(\phi_{\mid X} \times \operatorname{id}_{Y}\right)=A$.

Finally, we prove Lemma 3.1 By DG67, II 7.1.7], there is a discrete valuation ring $R$ and a morphism $\operatorname{Spec}(R) \rightarrow(X \times Y)^{2}$ such that the generic point maps into $\Sigma_{2}$ and the closed point maps to $(z, z)$. By extending $R$, we may assume that $R$ is of the form $\Omega[[t]]$ where $\Omega$ is a field.

Let us denote the first and the second components of the map by

$$
z_{i}(t)=\left(\boldsymbol{x}_{i}(t), y_{i}(t)\right): \operatorname{Spec}(\Omega[[t]]) \rightarrow X \times Y, \quad i=1,2 ;
$$

then we have in particular $z_{1}(0)=z_{2}(0)=z$ in $(X \times Y)(\Omega)$.

Denote the coordinates of $\boldsymbol{x}_{i}(t)$ in $\mathbb{A}^{N}$ by

$$
\begin{aligned}
\boldsymbol{x}_{i}(t) & =\left(x_{i, 1}(t), \ldots, x_{i, N}(t)\right), \quad \text { each of which are power series: } \\
x_{i, j}(t) & =\sum_{h \geq 0} x_{i, j}^{(h)} t^{h} \in \Omega[[t]] .
\end{aligned}
$$

Write $\boldsymbol{x}_{i}^{(h)}:=\left(x_{i, 1}^{(h)}, \ldots, x_{i, N}^{(h)}\right) \in \Omega^{N}$.

Since the generic point of $\operatorname{Spec}(\Omega[[t]])$ maps into $\Sigma_{2}$, we have $y_{1}(t)=y_{2}(t)$ in $Y(\Omega[[t]])$ and

$$
\boldsymbol{x}_{1}(t) \neq \boldsymbol{x}_{2}(t), \quad \delta\left(\boldsymbol{x}_{1}(t)-\boldsymbol{x}_{2}(t)\right) \in V(\phi)_{\Omega((t))} .
$$

In particular $\delta\left(\boldsymbol{x}_{1}^{(h)}-\boldsymbol{x}_{2}^{(h)}\right) \in V(\phi)_{\Omega}$ for all $h$ such that the vector $\boldsymbol{x}_{1}^{(h)}-\boldsymbol{x}_{2}^{(h)}$ is non-zero (otherwise the left hand side does not make sense).

Let $h_{0} \geq 1$ be the integer such that the vectors $\boldsymbol{x}_{1}(t)$ and $\boldsymbol{x}_{2}(t)$ satisfy $\boldsymbol{x}_{1}(t) \equiv \boldsymbol{x}_{2}(t)$ modulo $t^{h_{0}}$ and $\boldsymbol{x}_{1}(t) \not \equiv \boldsymbol{x}_{2}(t)$ modulo $t^{h_{0}+1}$. We claim that the first non-vanishing vector $\boldsymbol{x}_{1}^{\left(h_{0}\right)}-\boldsymbol{x}_{2}^{\left(h_{0}\right)} \in \Omega^{N}$ belongs to the subspace $T_{X, x} \otimes_{k(x)}$ $\Omega$.

Let $f\left(x_{1}, \ldots, x_{N}\right) \in \mathcal{O}\left(\mathbb{A}^{N}\right)$ be any function belonging to the defining ideal for $X$. We have $f\left(\boldsymbol{x}_{i}(t)\right)=0$ for $i=1,2$ so that

$$
f\left(\boldsymbol{x}_{1}(t)\right)-f\left(\boldsymbol{x}_{2}(t)\right)=0 \quad \text { in } \Omega[[t]] .
$$


Denote the coordinates of the point $\boldsymbol{x}_{1}(0)=\boldsymbol{x}_{2}(0)=x$ by $\left(x_{1}(0), \ldots, x_{N}(0)\right)$. Note that the functions $x_{i, j}(t)-x_{j}(0)$ are divisible by $t$ in $\Omega[[t]]$. Expand the function $f$ with center at $x$, and isolate the degree 1 terms:

$$
f=\left(\sum_{j=1}^{N} \frac{\partial f}{\partial x_{j}}(x) \cdot\left(x_{j}-x_{j}(0)\right)\right)+f_{\geq 2}
$$

where $f_{\geq 2}$ is the sum of degree $\geq 2$ terms with respect to $\left\{x_{j}-x_{j}(0)\right\}_{j}$. By the congruence $x_{i, j}(t)-x_{j}(0) \equiv 0$ modulo $t$ and $x_{1, j}(t) \equiv x_{2, j}(t)$ modulo $t^{h_{0}}$, the power series $f_{\geq 2}\left(\boldsymbol{x}_{1}(t)\right)-f_{\geq 2}\left(\boldsymbol{x}_{2}(t)\right)$ is divisible by $t^{h_{0}+1}$. It follows that formula (5) modulo $t^{h_{0}+1}$ reads:

$$
\sum_{j=1}^{N} \frac{\partial f}{\partial x_{j}}(x) \cdot\left(x_{1, j}^{\left(h_{0}\right)}-x_{2, j}^{\left(h_{0}\right)}\right) t^{h_{0}} \equiv 0 \quad \bmod t^{h_{0}+1},
$$

which precisely means $\left(d f_{x}\right)\left(\boldsymbol{x}_{1}^{\left(h_{0}\right)}-\boldsymbol{x}_{2}^{\left(h_{0}\right)}\right)=0$ in $\Omega$. Since this holds for all functions $f$ which vanish on $X$, the vector $\boldsymbol{x}_{1}^{\left(h_{0}\right)}-\boldsymbol{x}_{2}^{\left(h_{0}\right)}$ is tangent to $X$.

\section{Noether's normalization lemma over a Dedekind base}

The following is a variant of [Lev06, Th. 10.2.2] where he considers $X$ smooth over an arbitrary noetherian scheme $B$. In the next section, it will be applied to the situation where we are given a principal effective Cartier divisor $D \subset X$ defined by an equation $\pi: X \rightarrow \mathbb{A}^{1}=: B$.

Theorem 4.1. Let $B$ be the spectrum of a Dedekind domain and $p: X \rightarrow B$ be an equi-dimensional morphism (i.e., it is a morphism of finite type where every irreducible component of $X$ dominates $B$ and all fibers have the same dimension). Let $n$ be its fiber dimension.

Suppose we are given a point $x \in X$ and let $b=p(x) \in B$ be its image. Assume $k(b)$ is an infinite field. Then there exists Nisnevich neighborhoods $\left(X^{\prime}, x\right) \rightarrow(X, x)$ and $\left(B^{\prime}, b\right) \rightarrow(B, b)$, a commutative diagram

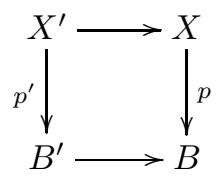

and a closed immersion $X^{\prime} \hookrightarrow \mathbb{A}_{B^{\prime}}^{N}$ for some $N \geq 0$ such that if we denote by $\bar{X}^{\prime}$ its closure in $\mathbb{P}_{B^{\prime}}^{N}$, then $X_{b}^{\prime}$ is dense in $\left(\bar{X}^{\prime}\right)_{b}$.

Consequently, after possibly replacing $B$ by a dense open subset, we can find a matrix $\phi \in M_{N \times n}^{*}(B)$ such that $\phi_{\mid X^{\prime}}: X^{\prime} \rightarrow \mathbb{A}_{B^{\prime}}^{N}$ is finite and surjective. 
In the proof below, given a map of schemes $X \rightarrow B$ and a property $\mathbf{P}$ of schemes (or of schemes and its subsets, when some subsets of $X$ are also given), we will say $\mathbf{P}$ is true fiberwise over $B$ to mean that the property $\mathbf{P}$ holds for every fiber of the morphism.

Many of the properties appearing below are such that if the property holds for the fiber over a point $b \in B$, then it holds for all fibers over the points in an open neighborhood of $b$. In this case, if we arrange that the property be true for the fiber over a given $b$ and we replace $B$ by such an open subset $B_{1}$, then the property becomes true fiberwise over $B_{1}$.

Proof. If $n=0$, this follows from a version of Hensel's lemma. We proceed by induction on $n$, and let us assume $n \geq 1$ in what follows.

Embed $X$ into a projective space $\mathbb{P}_{B}^{N}$ as a locally closed subscheme, and take its (reduced) closure $\bar{X}$. Since $B$ is Dedekind, $\bar{X}$ is still equi-dimensional over $B$ because every injective ring homomorphism from a Dedekind domain into an integral domain is flat.

[This is the only point where we use the assumption that $B$ is Dedekind, except that it is of course built in the induction step as well. Probably there are many special cases where this equi-dimensionality can be verified for a more general base $B$.]

Note that $X$ is not necessarily fiberwise dense in $\bar{X}$ over $B$ because there can be an irreducible component of $\bar{X}_{b}$ contained in $\bar{X} \backslash X$, which is the main difficulty we are going to address.

Possibly after shrinking $B$ to an open neighborhood $B_{1}$ of $b$, we can choose a tuple of sections $\psi=\left(\psi_{1}, \ldots, \psi_{n}\right) \in \Gamma\left(\mathbb{P}_{B_{1}}^{N}, \mathcal{O}(1)\right)^{n}$, which forms part of a basis for $\Gamma\left(\mathbb{P}_{B_{1}}^{N}, \mathcal{O}(1)\right)$, such that their common zero-locus $V(\psi)$ does not meet $x$, and meets $X_{1}:=X \times_{B} B_{1}$ fiberwise properly over $B_{1}$, so that their intersection is finite and surjective over $B_{1}$. Put $X_{2}:=\left(X \times_{B} B_{1}\right) \backslash V(\psi)$, which is an open neighborhood of $x$.

Let $\tilde{\mathbb{P}}_{B_{1}}^{N} \stackrel{\pi}{\rightarrow} \mathbb{P}_{B_{1}}^{N}$ be the blow-up along $V(\psi)$. Denote by $\tilde{X}_{1}$ the strict transform of $\bar{X}_{1}$. It actually turns out that $\tilde{X}_{1}=\pi^{-1}\left(\bar{X}_{1}\right)$, but we will only need the obvious inclusion $\tilde{X}_{1} \subset \pi^{-1}\left(\bar{X}_{1}\right)$. The rational map $\mathbb{P}_{B_{1}}^{N} \rightarrow \mathbb{P}_{B_{1}}^{n-1}$ : $\left[X_{0}: \cdots: X_{N}\right] \mapsto\left[\psi_{1}(\boldsymbol{X}): \cdots: \psi_{n}(\boldsymbol{X})\right]$ gives a well-defined smooth morphism $\psi: \tilde{\mathbb{P}}_{B_{1}}^{N} \rightarrow \mathbb{P}_{B_{1}}^{n-1}$.

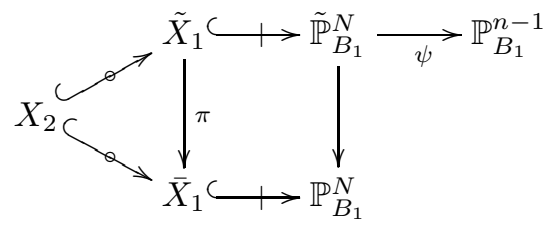

Some observations on this map are in order. Given a point $y=\left[\eta_{1}: \cdots\right.$ : $\left.\eta_{n}\right]: \operatorname{Spec}(\Omega) \rightarrow \mathbb{P}_{B_{1}}^{n-1}(\Omega$ is a field), the fiber of $\psi$ at $y$ is identified with the linear subscheme $V(y)$ of $\mathbb{P}_{\Omega}^{N}$ defined by the equations $\eta_{j} \psi_{k}\left(X_{0}, \ldots, X_{N}\right)=$ $\eta_{k} \psi_{j}\left(X_{0}, \ldots, X_{N}\right)(j, k \in\{1, \ldots, n\})$. If we choose an index $j$ with $\eta_{j} \neq 0$, a 
minimal set of equations can be chosen as

$$
\eta_{j} \psi_{k}\left(X_{0}, \ldots, X_{N}\right)=\eta_{k} \psi_{j}\left(X_{0}, \ldots, X_{N}\right) \quad k \in\{1, \ldots, n\} \backslash\{j\} .
$$

It contains $V(\psi)_{\Omega}$ and isomorphic to $\mathbb{P}_{\Omega}^{N-n+1}$.

Let $E(\psi) \subset \tilde{\mathbb{P}}_{B_{1}}^{N}$ be the exceptional divisor and $\pi: E(\psi) \rightarrow V(\psi)$ the restriction of the blow-up map.

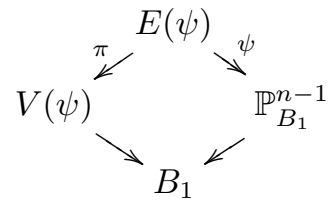

It turns out that this square is cartesian.

Now, let us observe that the restriction $\psi_{\mid \tilde{X}_{1}}: \tilde{X}_{1} \rightarrow \mathbb{P}_{B_{1}}^{n-1}$ is equi-dimensional of fiber dimension 1. Indeed, given a point $y \in \mathbb{P}_{B_{1}}^{n-1}(\Omega)$, consider $\left(\bar{X}_{1}\right)_{\Omega}:=$ $\bar{X}_{1} \times_{B_{1}} \Omega \subset \mathbb{P}_{\Omega}^{N}$ which has dimension $n$. The fiber of $\psi_{\mid \tilde{X}_{1}}$ over $y$ is contained in the intersection $\left(\bar{X}_{1}\right)_{\Omega} \cap V(y)$ in $\mathbb{P}_{\Omega}^{N}$. We know that $V(y)$ contains $V(\psi)_{\Omega}$ as a linear subscheme of codimension 1. Every irreducible component $Y_{\alpha}$ of $\left(\bar{X}_{1}\right)_{\Omega} \cap V(y)$ meets $V(\psi)_{\Omega}$ because it is the intersection in a projective space, and we chose $\psi$ so that the intersection $Y_{\alpha} \cap V(\psi)_{\Omega}$ has dimension 0. Therefore we conclude that $Y_{\alpha}$ has dimension 1 .

Set $T:=\mathbb{P}_{B_{1}}^{n-1}$ and $t:=\psi(x) \in \mathbb{P}_{B_{1}}^{n-1}$. Choose any projective embedding $\tilde{X}_{1} \hookrightarrow \mathbb{P}_{T}^{N_{2}}$. Set $\left(\tilde{X}_{1}\right)_{t}:=\tilde{X}_{1} \times_{T} t$ which is in $\mathbb{P}_{t}^{N_{2}}$. Also, set $\left(X_{2}\right)_{t}:=X_{2} \times_{T} t$ which is an open subset of $\left(\tilde{X}_{1}\right)_{t}$. There is a hypersurface $H_{t}$ in $\mathbb{P}_{t}^{N_{2}}$ satisfying the next three conditions. [Indeed, there exist hypersurfaces satisfying (i) in some high degree; a general one among such (of any fixed degree) satisfies (ii) and (iii).]

(i) [In case $x$ is a closed point of $\left(X_{2}\right)_{t}$ : $] H_{t}$ contains $x$.

(ii) $\left(\tilde{X}_{1}\right)_{t}$ and $H_{t}$ meet properly in $\mathbb{P}_{t}^{N_{2}}$.

(iii) [Let $\left(X_{2}\right)_{t}^{-}$be the closure of $\left(X_{2}\right)_{t}$ in $\mathbb{P}_{t}^{N_{2}}$. Then $\left(X_{2}\right)_{t}^{-} \backslash\left(X_{2}\right)_{t}$ is a finite set. That being said:] $H_{t}$ does not meet $\left(X_{2}\right)_{t}^{-} \backslash\left(X_{2}\right)_{t}$.

Let $T^{\mathrm{h}} \rightarrow T$ be the henselization of $T$ at $t$. Let $H_{T^{\mathrm{h}}} \subset \mathbb{P}_{T^{\mathrm{h}}}^{N_{2}}$ be any flat family of hypersurfaces that specializes to $H_{t}$. Base change by $T^{\mathrm{h}} \rightarrow T$ will be denoted by the subscript $(-)_{T^{\mathrm{h}}}$. The closed subscheme $D_{T^{\mathrm{h}}}:=H_{T^{\mathrm{h}}} \times_{\mathbb{P}_{T}^{N_{2}}} \tilde{X}_{1}$ of $\left(\tilde{X}_{1}\right)_{T^{\mathrm{h}}}$ is an effective Cartier divisor because $\left(\tilde{X}_{1}\right)_{T^{\mathrm{h}}}$ is reduced (hence without embedded components). By condition (ii), the scheme $D_{T^{\mathrm{h}}}$ is the disjoint union of its local components finite over $T^{\mathrm{h}}$; in particular, it decomposes as

$$
D_{T^{\mathrm{h}}}=\mathcal{D}_{T^{\mathrm{h}}} \sqcup \mathcal{D}_{T^{\mathrm{h}}}^{\prime}
$$


where $\mathcal{D}_{T^{\mathrm{h}}}$ has closed points in the open set $\left(X_{2}\right)_{t}$ (and hence it is contained in $\left.\left(X_{2}\right)_{T^{\mathrm{h}}}\right)$ and $\mathcal{D}_{T^{\mathrm{h}}}^{\prime}$ is disjoint from $\left(X_{2}\right)_{t}$.

By the usual limit argument as in [DG67, $\mathrm{IV}_{2} \S 8$ ], there is an affine Nisnevich neighborhood $T_{3} \rightarrow T$ of $t$ such that: we can take a hypersurface $H_{T_{3}}$ such that $H_{T^{\mathrm{h}}}=H_{T_{3}} \times_{T_{3}} T^{\mathrm{h}}$; the decomposition of $D_{T^{\mathrm{h}}}$ comes from a decomposition $D_{T_{3}}=\mathcal{D} \sqcup \mathcal{D}^{\prime}$; and the conditions stated in the previous paragraph become true already after the base change $T_{3} \rightarrow T$. Let us denote $\tilde{X}_{3}:=\tilde{X}_{1} \times_{T} T_{3}$ and $X_{3}:=X_{2} \times_{T} T_{3}$. Then we have:

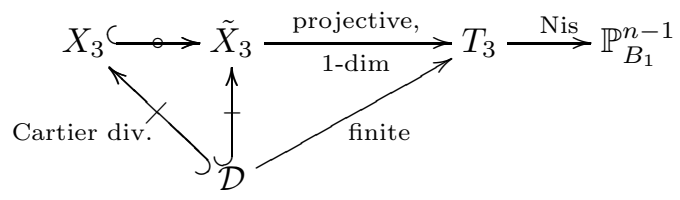

For integers $m$, we can consider the line bundles $\mathcal{O}_{\tilde{X}_{3}}(m \mathcal{D})$ on $\tilde{X}_{3}$. We claim that for any sufficiently large $m>0$, the restriction map

$$
\Gamma\left(\tilde{X}_{3}, \mathcal{O}_{\tilde{X}_{3}}(m \mathcal{D})\right) \rightarrow \Gamma\left(\mathcal{D}, \mathcal{O}_{\mathcal{D}}(m \mathcal{D})\right)
$$

is surjective. Indeed, we have an exact sequence $0 \rightarrow \mathcal{O}_{\tilde{X}_{3}}(-\mathcal{D}) \rightarrow \mathcal{O}_{\tilde{X}_{3}} \rightarrow$ $\mathcal{O}_{\mathcal{D}} \rightarrow 0$ by definitions, which gives exact sequences

$$
0 \rightarrow \mathcal{O}_{\tilde{X}_{3}}(m \mathcal{D}) \rightarrow \mathcal{O}_{\tilde{X}_{3}}((m+1) \mathcal{D}) \rightarrow \mathcal{O}_{\mathcal{D}}((m+1) \mathcal{D}) \rightarrow 0 .
$$

Since $\mathcal{D}$ is an affine scheme, we have $H^{1}\left(\mathcal{D}, \mathcal{O}_{\mathcal{D}}((m+1) \mathcal{D})\right)=0$. Therefore we have surjections

$$
\begin{aligned}
H^{1}\left(\tilde{X}_{3}, \mathcal{O}_{\tilde{X}_{3}}(m \mathcal{D})\right) & \rightarrow H^{1}\left(\tilde{X}_{3}, \mathcal{O}_{\tilde{X}_{3}}((m+1) \mathcal{D})\right) \rightarrow \ldots \\
& \rightarrow H^{1}\left(\tilde{X}_{3}, \mathcal{O}_{\tilde{X}_{3}}((m+k) \mathcal{D})\right) \rightarrow \ldots
\end{aligned}
$$

Since $M_{m}:=H^{1}\left(\tilde{X}_{3}, \mathcal{O}_{\tilde{X}_{3}}(m \mathcal{D})\right)$ is a finitely generated $\Gamma\left(T_{3}, \mathcal{O}_{T_{3}}\right)$-module, which is noetherian, the group $\operatorname{ker}\left(M_{m} \rightarrow M_{m+k}\right)$ stabilizes as $k$ tends to $\infty$. Therefore $M_{m+k} \rightarrow M_{m+k+1}$ is an isomorphism for any sufficiently large $k$. It follows that in the cohomology long exact sequence associated to the short exact sequence (7) with the index shifted:

$$
H^{0}\left(\tilde{X}_{3}, \mathcal{O}_{\tilde{X}_{3}}(m \mathcal{D})\right) \rightarrow H^{0}\left(\mathcal{D}, \mathcal{O}_{\mathcal{D}}(m \mathcal{D})\right) \rightarrow M_{m-1} \rightarrow M_{m},
$$

the last map is bijective for any sufficiently large $m$. We conclude that the map $H^{0}\left(\tilde{X}_{3}, \mathcal{O}_{\tilde{X}_{3}}(m \mathcal{D})\right) \rightarrow H^{0}\left(\mathcal{D}, \mathcal{O}_{\mathcal{D}}(m \mathcal{D})\right)$ is surjective.

By this surjectivity, we can find a section $s_{0} \in \Gamma\left(\tilde{X}_{3}, \mathcal{O}_{\tilde{X}_{3}}(m \mathcal{D})\right)$ which maps to a nowhere vanishing section of $\Gamma\left(\mathcal{D}, \mathcal{O}_{\mathcal{D}}(m \mathcal{D})\right) \cong \Gamma\left(\mathcal{D}, \mathcal{O}_{\mathcal{D}}\right)$. Let $s_{1}: \mathcal{O}_{\tilde{X}_{3}} \rightarrow$ $\mathcal{O}_{\tilde{X}_{3}}(m \mathcal{D})$ be the canonical inclusion. Since the zero-loci of $s_{0}$ and $s_{1}$ are disjoint (one is away from $\mathcal{D}$ and the other is $|\mathcal{D}|$ ), we have a well-defined morphism

$$
f:=\left(s_{0}: s_{1}\right): \tilde{X}_{3} \rightarrow \mathbb{P}_{T_{3}}^{1} .
$$


Let us consider its restriction to $\left(\tilde{X}_{3}\right)_{t}=\left(\tilde{X}_{1}\right)_{t}$ which is a projective curve over $k(t)$. The inverse image of $\infty \in \mathbb{P}_{t}^{1}$ is exactly $\left(\tilde{X}_{1}\right)_{t} \cap \mathcal{D}$. Let $Y_{\alpha}$ be any irreducible component of $\left(\tilde{X}_{1}\right)_{t}$. If it meets the open subset $\left(X_{3}\right)_{t}=\left(X_{2}\right)_{t}$, then $Y_{\alpha} \cap \mathcal{D}$ is a non-empty finite set by the definition of $\mathcal{D}$ (it is non-empty by condition (iii)); in this case $Y_{\alpha} \rightarrow \mathbb{P}_{t}^{1}$ is finite flat. To the contrary, if $Y_{\alpha}$ is away from $\left(X_{3}\right)_{t}$, we have $Y_{\alpha} \cap \mathcal{D}=\emptyset$; in this case $Y_{\alpha} \rightarrow \mathbb{P}_{t}^{1}$ is a constant map to a point.

The quasi-finite locus of a morphism is open [DG67, $\mathrm{IV}_{3}$ 13.1.4]. We can find an affine open neighborhood $T_{4} \subset T_{3}$ of $t$ such that $\mathcal{D}_{4}:=\mathcal{D}_{T_{4}}$ is contained in the quasi-finite locus of $f$. Let $f_{4}: \tilde{X}_{4} \rightarrow \mathbb{P}_{T_{4}}^{1}$ be the base change $f \times_{T_{3}} T_{4}$ and let $X_{4} \subset X_{3}$ be the quasi-finite locus of $f_{\mid X_{3} \times_{T_{3}} T_{4}}$.

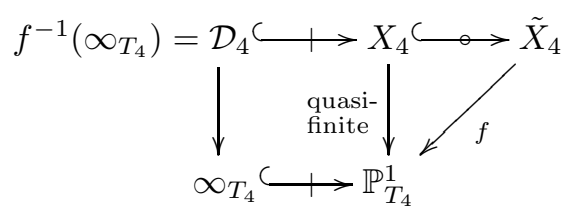

Then the subset $W_{4}:=f\left(\tilde{X}_{4} \backslash X_{4}\right) \subset \mathbb{P}_{T_{4}}^{1}$ is proper over $T_{4}$ and is contained in $\mathbb{P}_{T_{4}}^{1} \backslash \infty_{T_{4}}=\mathbb{A}_{T_{4}}^{1}$; therefore it is finite over $T_{4}$. The induced map from $\tilde{X}_{4} \backslash f^{-1}\left(W_{4}\right)$ to $\mathbb{P}_{T_{4}}^{1} \backslash W_{4}$ is finite because it is proper and quasi-finite (as $f$ is quasi-finite on $\left.X_{4}\right)$. Note that $\tilde{X}_{4} \backslash f^{-1}\left(W_{4}\right)$ contains $x$ by condition (i).

Now, by induction on $n$, there are Nisnevich neighborhoods $B_{5} \rightarrow B_{1}$ of $b$ and $T_{5} \rightarrow T_{4} \times_{B_{1}} B_{5}$ of $t$ such that there is a projective compactification $T_{5} \hookrightarrow \bar{T}_{5}$ which is fiberwise dense over $B_{5}$. Base change of $T_{4}$-schemes by $T_{5} \rightarrow T_{4}$ will be indicated by replacement of the number.

Take any factorization of $f$ of the form $\tilde{X}_{5} \hookrightarrow \mathbb{P}_{T_{5}}^{N_{5}} \times_{T_{5}} \mathbb{P}_{T_{5}}^{1} \rightarrow \mathbb{P}_{T_{5}}^{1}$ and denote by $\bar{X}_{5}$ the reduced closure of $\tilde{X}_{5}$ in $\mathbb{P}_{\bar{T}_{5}}^{N_{5}} \times_{\bar{T}_{5}} \mathbb{P}_{\bar{T}_{5}}^{1}$. We have the following diagram where every square is cartesian:

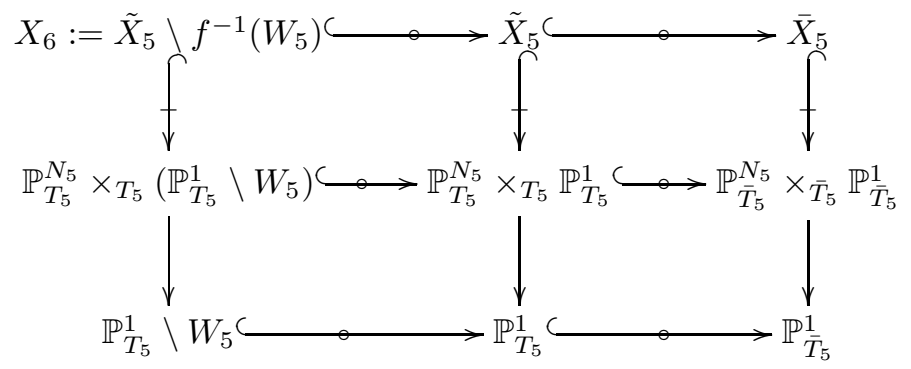

Take the Stein factorization [DG67, III $\left._{1} \S 4.3\right]$ of the composite map $\bar{f}_{5}: \bar{X}_{5} \rightarrow$ $\mathbb{P}_{\bar{T}_{5}}^{1}$ :

$$
\bar{X}_{5} \rightarrow \bar{X}_{6} \stackrel{\text { finite }}{\longrightarrow} \mathbb{P}_{\bar{T}_{5}}^{1}
$$

so that the first map $\bar{X}_{5} \rightarrow \bar{X}_{6}$ has geometrically connected fibers. Since $\bar{f}_{5}$ is 
already finite over the open set $\mathbb{P}_{T_{5}}^{1} \backslash W_{5}$ of the target, we know $\bar{X}_{6} \times_{\mathbb{P}_{T_{5}}^{1}}\left(\mathbb{P}_{T_{5}}^{1} \backslash W_{5}\right)$ is isomorphic to $X_{6}:=\tilde{X}_{5} \backslash f^{-1}\left(W_{5}\right)$.

Now we claim that the open immersion $X_{6}{ }^{\oplus} \bar{X}_{6}$ is fiberwise dense over $B_{5}$. First, note that $\bar{T}_{5}$ and $\bar{X}_{6}$ are equi-dimensional over $B_{5}$ with fiber dimensions $n-1$ and $n$ by the same reasoning as in the second paragraph of this proof (using the fact that $B_{5}$ is Dedekind). Let $b^{\prime} \in B_{5}$ be any point and $Y_{\beta} \subset\left(\bar{X}_{6}\right)_{b^{\prime}}$ be any irreducible component of the fiber. We have to prove that $Y_{\beta}$ meets the open set $X_{6}$.

The composition $Y_{\beta} \hookrightarrow\left(\bar{X}_{6}\right)_{b^{\prime}} \rightarrow\left(\mathbb{P}_{\bar{T}_{5}}^{1}\right)_{b^{\prime}}$ is a finite morphism of $n$-dimensional $k\left(b^{\prime}\right)$-schemes, and so it is a surjection to an irreducible component. The open set $\left(\mathbb{P}_{T_{5}}^{1} \backslash W_{5}\right)_{b^{\prime}}$ of the target is dense because $\left(T_{5}\right)_{b^{\prime}} \subset\left(\bar{T}_{5}\right)_{b^{\prime}}$ is dense and $W_{5}$ is finite over $T_{5}$. Therefore its inverse image to $Y_{\beta}$, which is exactly $Y_{\beta} \cap X_{6}$, is non-empty. This verifies that $X_{6} \subset \bar{X}_{6}$ is fiberwise dense over $B_{5}$.

It remains to find claimed affine and projective embeddings. The scheme $\bar{X}_{6}$ is projective over $B_{5}$ because the morphisms $\bar{X}_{6} \rightarrow \bar{T}_{5}$ and $\bar{T}_{5} \rightarrow \bar{B}_{5}$ are, so take any closed immersion $\bar{X}_{6} \hookrightarrow \mathbb{P}_{B_{5}}^{N_{6}}$. Since the ideal of the closed subscheme $\left(\bar{X}_{6} \backslash X_{6}\right)_{\text {red }}$ is generated by global sections after sufficient Serre twist, there exists a flat family of hypersurfaces $H_{B_{5}} \subset \mathbb{P}_{B_{5}}^{N_{6}}$ containing $\bar{X}_{6} \backslash X_{6}$ and such that its fiber $H_{b}$ over $b$ meets $\left(\bar{X}_{6}\right)_{b}$ properly in $\mathbb{P}_{b}^{N_{5}}$. Also, we require that $H_{b}$ does not contain $x$. There is an affine open neighborhood $B_{7} \rightarrow B_{5}$ of $b$ such that the base change $H_{B_{7}}$ mees $\bar{X}_{7}:=\bar{X}_{6} \times_{B_{5}} B_{7}$ fiberwise properly over $B_{7}$.

Let $d$ be the degree of $H_{B_{7}}$. Then via the $d$-fold Veronese embedding $\mathbb{P}_{B_{7}}^{N_{5}} \leftrightarrow \mathbb{P}_{B_{7}}^{N_{7}}$ followed by a linear automorphism, $H_{B_{7}}$ is the restriction of the infinity-hyperplane of $\mathbb{P}_{B_{7}}^{N_{7}}$. Put $X_{7}:=\bar{X}_{7} \backslash H_{B_{7}}$ and consider $\bar{X}_{7}$ as a closed subscheme of $\mathbb{P}_{B_{7}}^{N_{7}}$. Then the cartesian diagram

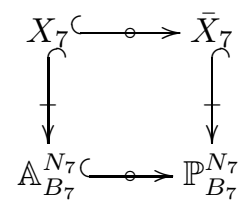

verifies our assertion.

\section{General case}

Definitioin 5.1. Let $\mathcal{C}$ be a family of constructible subsets of an equi-dimensional scheme $X$ and $q \geq 0$ be an integer. Define the shifted family $\mathcal{C}[q]$ by $(\mathcal{C}[q])_{d}:=$ $\mathcal{C}_{d-q}$ if $d<\operatorname{dim}(X)$ and $:=X$ if $d \geq \operatorname{dim}(X)$. 
For example $\mathcal{C}[1]$ is the following (write $n:=\operatorname{dim}(X)$ ):

$$
\begin{array}{lccccccccc} 
& & -1 & 0 & 1 & 2 & \ldots & \multicolumn{1}{l}{\operatorname{dim}(X)-1} & \operatorname{dim}(X) \\
\mathcal{C}: & \emptyset & \subseteq C_{0} \subseteq C_{1} \subseteq C_{2} \subseteq \ldots & \subseteq & C_{n-1} & \subseteq & X \\
\mathcal{C}[1]: & \emptyset & \subseteq & \emptyset & \subseteq C_{0} \subseteq C_{1} \subseteq \ldots & \subseteq & C_{n-2} & \subseteq & X
\end{array}
$$

Note that we have a chain law $(\mathcal{C}[p])[q]=\mathcal{C}[p+q]$ and $\mathcal{C}[n]$ is the trivial family.

We go back to the notation of Main Theorem 1.6 namely, we are given an effective Cartier divisor $D \subset X$ of an equi-dimensional $k$-scheme of dimension $n$ and a family $\mathcal{C}$ of constructible subsets of $X \backslash D$, and an equi-dimensional $k$-scheme $Y$. To prove that the inclusion $z_{\mathcal{C}}^{i}\left(-\times Y \mid D_{-} \times Y, \bullet\right) \subset z^{i}(-\times Y \mid$ $\left.D_{-} \times Y, \bullet\right)$ is a quasi-isomorphism on $X_{\text {Nis }}$, it suffices to show that the quotient complex $z_{\mathcal{C}[q]}^{i}\left(-\times Y \mid D_{-} \times Y, \bullet\right) / z_{\mathcal{C}[q-1]}^{i}$ is Nisnevich locally acyclic for $1 \leq q \leq n$, for then in the sequence below:

$$
z_{\mathcal{C}}^{i} \subset z_{\mathcal{C}[1]}^{i} \subset \ldots z_{\mathcal{C}[n]}^{i}=z^{i}\left(-\times Y \mid D_{-} \times Y, \bullet\right),
$$

every inclusion is a Nisnevich local quasi-isomorphism on $X$. Refreshing the notation $\mathcal{C}:=\mathcal{C}[q-1]$, it suffices to prove that the quotient complex $z_{\mathcal{C}[1]}^{i}(-\times Y \mid$ $\left.D_{-} \times Y, \bullet\right) / z_{\mathcal{C}}^{i}$ is locally acyclic.

Let us recall the following principle: To show that a given complex $Z$ • of abelian groups is acyclic, it suffices to show that for every finitely generated subcomplex $Z_{\bullet}^{\prime}$ (i.e., it has only finitely many non-trivial terms, each of which is a finitely generated abelian group), the inclusion $Z_{\bullet}^{\prime} \subset Z_{\bullet}$ induces the zero map on homology. This is because every homology class of $Z \bullet$ comes from some $Z_{\bullet}^{\prime}$.

Proof of Theorem 1.6. Since the assertion is local on $X$, we may assume that $X$ is affine and $D$ is a principal divisor defined by a function $\pi: X \rightarrow \mathbb{A}^{1}$. We take an arbitrary finite set $\left\{V_{\lambda} \subset X \times Y \times \square^{p(\lambda)}\right\}_{\lambda}$ of irreducible cycles belonging to $z_{\mathcal{C}[1]}^{i}(X \times Y \mid D \times Y, \bullet)$ which is closed under the passage from a cycle $V_{\lambda}$ to the irreducible components of its restriction to faces.

Consider the subcomplex

$$
\frac{z_{\mathcal{C}[1]}^{i \prime}}{z_{\mathcal{C}}^{i}} \quad \text { of } \quad \frac{z_{\mathcal{C}[1]}^{i}(X \times Y \mid D \times Y, \bullet)}{z_{\mathcal{C}}^{i}(X \times Y \mid D \times Y, \bullet)}
$$

generated by the cycles $\left\{V_{\lambda}\right\}_{\lambda}$; we have to find a Nisnevich neighborhood $X^{\prime} \rightarrow$ $X$ of a given point $x \in X$ such that the map (where $D^{\prime}:=D \times_{X} X^{\prime}$ )

$$
\frac{z_{\mathcal{C}[1]}^{i \prime}}{z_{\mathcal{C}}^{i}} \rightarrow \frac{z_{\mathcal{C}[1]}^{i}\left(X^{\prime} \times Y \mid D^{\prime} \times Y, \bullet\right)}{z_{\mathcal{C}}^{i}\left(X^{\prime} \times Y \mid D^{\prime} \times Y, \bullet\right)}
$$

induces the zero map on homology.

Consider all the subsets of the form $V_{\lambda} \cap\left(C_{d} \times Y \times \square^{p(\lambda)}\right)$, and write $S \subset X \backslash D$ for the finite set consisting of the projections of their generic points to $X$. 
Lemma 5.2. There exist an affine open neighborhood $X_{1}$ of $x$ in $X$ and a unit $f \in \mathcal{O}\left(X_{1}\right)^{*}$ such that the function $f \pi: X_{1} \rightarrow \mathbb{A}^{1}$ is smooth on the finite set $S \cap X_{1}$.

[The following proof would become simpler if $x$ is in the smooth locus of $X$.]

Let $X \hookrightarrow \mathbb{A}_{k}^{N}$ be any closed immersion and consider linear projections $\phi: \mathbb{A}^{N} \rightarrow$ $\mathbb{A}^{1}$. For a general $\phi$, the induced map $f_{0}:=\phi_{\mid X}: X \rightarrow \mathbb{A}_{k}^{1}$ is smooth at the points of $S$ (e.g. by 3.2 .2 ). Fix such an $f_{0}$. We are going to choose $f$ in the form

$$
f=f_{0}+a \quad(a \in k) .
$$

The morphism $f \pi$ is smooth at a given point $x_{i} \in X$ if and only if the induced $k\left(x_{i}\right)$-linear map [let $t$ be the coordinate for $\mathbb{A}_{k}^{1}$ ]

$$
(f \pi)^{*}: k\left(x_{i}\right) \cdot d t \rightarrow T_{x_{i}} X
$$

is injective. Here, the basis $d t$ is mapped to the next element of $T_{x_{i}} X$ :

$$
\begin{aligned}
d(f \pi)\left(x_{i}\right) & =\pi\left(x_{i}\right) \cdot(d f)\left(x_{i}\right)+f\left(x_{i}\right) \cdot(d \pi)\left(x_{i}\right) \\
& =\pi\left(x_{i}\right) \cdot\left(d f_{0}\right)\left(x_{i}\right)+\left(f_{0}\left(x_{i}\right)+a\right) \cdot(d \pi)\left(x_{i}\right) .
\end{aligned}
$$

Since the first term on the right hand side is non-zero if $x_{i} \in S$, this element is non-zero for all but possibly one value of $a \in k$. Since $S$ is a finite set, these elements are simultaneously non-zero for all $x_{i} \in S$ if $a \in k$ avoids a certain finite set.

Lastly, the function $f_{0}+a$ is invertible at each point of $S \cup\{x\}$ for a general $a \in k$. For such a general choice of $a \in k$, set $X_{1}:=f^{-1}\left(\mathbb{A}^{1} \backslash\{0\}\right)$. This completes the proof of Lemma 5.2 .

Returning to the proof of Theorem 1.6, let $B \rightarrow \mathbb{A}_{k}^{1}$ be the henselization at the origin. We apply Theorem 4.1 to the equi-dimensional morphism $f \pi: X_{1} \rightarrow$ $\mathbb{A}_{k}^{1}$ of fiber dimension $n-1$ to find a Nisnevich neighborhood $X^{\prime} \rightarrow X_{1} \times_{\mathbb{A}^{1}} B$ of $x$ and an embedding $X^{\prime} \hookrightarrow \mathbb{A}_{B}^{N}$ such that the following is true: If $\bar{X}^{\prime} \subset \mathbb{P}_{B}^{N}$ is the closure of $X^{\prime}$ in the projective space, then its intersection with the infinityhyperplane $\bar{X}^{\prime} \cap\left(\mathbb{P}_{B}^{N} \backslash \mathbb{A}_{B}^{N}\right)$ is equi-dimensional over $B$ of fiber dimension $n-2$. Base-change from $X$ to $X^{\prime}$ shall be indicated by primes $(-)^{\prime}$ : For example $D^{\prime}:=D \times_{X} X^{\prime}$ and $C_{d}^{\prime}=C_{d} \times_{X} X^{\prime}$.

It follows that there is a dense open subset $U$ of $\left(M_{N \times(n-1)}\right)_{\{0\}}$ such that if a matrix $\phi \in M_{N \times(n-1)}(B)$ specializes to a matrix in $U$, then the induced map $\phi_{\mid X^{\prime}}: X^{\prime} \rightarrow \mathbb{A}_{B}^{n-1}$ is finite and surjective (hence flat on the smooth locus of $X^{\prime}$ DG67, $\left.\left.\mathrm{IV}_{2} 6.1 .5\right]\right)$. In particular, pull-back and push-forward by $\phi_{\mid X^{\prime}}$ preserve the modulus condition of cycles, where $X^{\prime}$ is equipped with the modulus $D^{\prime}$ and $\mathbb{A}_{B}^{n-1}$ with $\mathbb{A}_{\{0\}}^{n-1}$. For such a $\phi$ and each of $V_{\lambda}$, we may consider the cycle $\left(\phi_{\mid X^{\prime}}^{*} \phi_{\mid X^{\prime} *}\left(V_{\lambda}^{\prime}\right)\right)-\left(V_{\lambda}^{\prime}\right)$ which is an element of $z^{i}\left(X^{\prime} \times Y \mid D^{\prime} \times Y, p(\lambda)\right)$. We denote it by $\phi^{*} \phi_{*} V_{\lambda}^{\prime}-V_{\lambda}^{\prime}$ for short. Below, when we say that a general $\phi \in$ $M_{N \times(n-1)}^{*}$ has some property, we mean that there is a dense open subset $U^{\prime}$ of $\left(M_{N \times(n-1)}^{*}\right)_{\operatorname{Frac}(B)}$ such that every $\phi \in U^{\prime}$ specializing into $U$ has the property. 
(In this case, such a $\phi$ exists because the matrices in $\left(M_{N \times(n-1)}^{*}\right)_{\operatorname{Frac}(B)}$ which specialize into $U$ form a dense subset.)

By the usual limit argument, the cycle complex $z^{i}\left(X^{\prime} \times Y \mid D^{\prime} \times Y, \bullet\right)$ is the direct limit of complexes $z^{i}\left(X_{\nu} \times Y \mid D_{\nu} \times Y, \bullet\right)$ where $X_{\nu} \rightarrow X$ runs through certain Nisnevich neighborhoods of $x$ of finite type. For a family $\mathcal{C}$ of constructible subsets of $X \backslash D$, we write $z_{\mathcal{C}}^{i}\left(X^{\prime} \times Y \mid D^{\prime} \times Y, \bullet\right)$ for the limit of the subcomplexes $z_{\mathcal{C}}^{i}\left(X_{\nu} \times Y \mid D_{\nu} \times Y, \bullet\right)$ (we are omitting the pull-back notation for $\mathcal{C}$ as usual). In fact, the literal definition of that group does not make sense because $X^{\prime} \backslash D^{\prime}$ has dimension $n-1$. The interested reader will find that our $z_{\mathcal{C}}^{i}\left(X^{\prime} \times Y \mid D^{\prime} \times Y, \bullet\right)$ is equal to the literally defined $z_{\mathcal{C}[-1]^{\prime}}^{i}\left(X^{\prime} \times Y \mid D^{\prime} \times Y, \bullet\right)$. We adopt the same convention with $z_{\phi_{*} \mathcal{C}}^{i}\left(\mathbb{A}_{B}^{n-1} \times Y \mid \mathbb{A}_{\{0\}}^{n-1} \times Y, \bullet\right)$ appearing below.

By 3.2 .3 applied to the scheme $X^{\prime} \backslash D^{\prime}$ over the field $\operatorname{Frac}(B)$, we know that a general $\phi \in M_{N \times(n-1)}^{*}$ has the property that the support of the cycle $\phi^{*} \phi_{*} V_{\lambda}^{\prime}-V_{\lambda}^{\prime}$ is $\phi^{+}\left(V_{\lambda}^{\prime}\right)$.

Thanks to Lemma 5.2 we may apply 3.2 .4 to subsets $V_{\lambda}^{\prime}$ and $W:=C_{d}^{\prime}$ of $X^{\prime} \backslash D^{\prime}$. It follows that a general $\phi \in M_{N \times(n-1)}^{*}$ satisfies the following for all $\lambda$ and $d$ :

$$
\begin{aligned}
& \operatorname{dim}\left(\left|\phi^{*} \phi_{*} V_{\lambda}^{\prime}-V_{\lambda}^{\prime}\right| \cap\left(C_{d}^{\prime} \times Y \times \square^{p(\lambda)}\right)\right) \\
& \quad \leq \max \left\{\operatorname{dim}\left(V_{\lambda}^{\prime} \cap\left(C_{d}^{\prime} \times Y \times \square^{p(\lambda)}\right)\right)-1, \operatorname{dim}\left(V_{\lambda}^{\prime}\right)+\operatorname{dim}\left(C_{d}^{\prime}\right)-\operatorname{dim}\left(X^{\prime}\right)\right\} .
\end{aligned}
$$

This formula precisely means that $\phi^{*} \phi_{*} V_{\lambda}^{\prime}-V_{\lambda}^{\prime}$ is in the smaller subcomplex $z_{\mathcal{C}}^{i}\left(X^{\prime} \times Y \mid D^{\prime} \times Y, \bullet\right)$. In particular, we deduce that $\phi^{*} \phi_{*} V_{\lambda}^{\prime}$ is in $z_{\mathcal{C}[1]}^{i}\left(X^{\prime} \times\right.$ $\left.Y \mid D^{\prime} \times Y, \bullet\right)$. Hence, we have the equality

$$
\phi^{*} \phi_{*} V_{\lambda}^{\prime}=V_{\lambda}^{\prime} \quad \text { in } \quad \frac{z_{\mathcal{C}[1]}^{i}\left(X^{\prime} \times Y \mid D^{\prime} \times Y, \bullet\right)}{z_{\mathcal{C}}^{i}\left(X^{\prime} \times Y \mid D^{\prime} \times Y, \bullet\right)} .
$$

It also follows that $\phi_{*} V_{\lambda}^{\prime}$ is in $z_{\phi_{*} \mathcal{C}[1]}^{i}\left(\mathbb{A}_{B}^{n-1} \times Y \mid \mathbb{A}_{\{0\}}^{n-1} \times Y, \bullet\right)$. This is because the subset $\left(\phi_{*} V_{\lambda}^{\prime}\right) \cap\left(\phi\left(C_{d}\right) \times Y \times \square^{p(\lambda)}\right)$ is equal to $\phi\left(\phi^{*} \phi_{*} V_{\lambda}^{\prime} \cap\left(C_{d} \times Y \times \square^{p(\lambda)}\right)\right)$ by the usual 'projection formula' of subsets. If $V_{\lambda}$ already happens to be in the smaller subcomplex $z_{\mathcal{C}}^{i}(X \times Y \mid D \times Y, \bullet)$, the same reasoning gives:

$$
\begin{array}{ll} 
& \phi^{*} \phi_{*} V_{\lambda}^{\prime} \in z_{\mathcal{C}}^{i}\left(X^{\prime} \times Y \mid D^{\prime} \times Y, \bullet\right) \\
\text { and } \quad & \phi_{*} V_{\lambda}^{\prime} \in z_{\phi_{*}}^{i} \mathcal{C}\left(\mathbb{A}_{B}^{n-1} \times Y \mid \mathbb{A}_{\{0\}}^{n-1} \times Y, \bullet\right) .
\end{array}
$$

Therefore, the first map of the next sequence is well-defined:

$$
\frac{z_{\mathcal{C}[1]}^{i \prime}}{z_{\mathcal{C}}^{i}} \stackrel{\phi_{*}}{\longrightarrow} \frac{z_{\phi_{*} \mathcal{C}[1]}^{i}\left(\mathbb{A}_{B}^{n-1} \times Y \mid \mathbb{A}_{\{0\}}^{n-1} \times Y, \bullet\right)}{z_{\phi_{*} \mathcal{C}}^{i}} \stackrel{\phi^{*}}{\longrightarrow} \frac{z_{\mathcal{C}[1]}^{i}\left(X^{\prime} \times Y \mid D^{\prime} \times Y, \bullet\right)}{z_{\mathcal{C}}^{i}}
$$

and the composite is equal to the pull-back map by $X^{\prime} \rightarrow X$. On the other hand, since the middle complex is acyclic by Theorem 2.1 the composite induces the zero map on homology. This completes the proof of Theorem 1.6. 
For Theorem 1.7 (the case of additive higher Chow), we proceed similarly using affine embedding $X \hookrightarrow \mathbb{A}_{k}^{N}$ and general linear projection $\mathbb{A}_{k}^{N} \rightarrow \mathbb{A}_{k}^{\operatorname{dim}(X)}$, and use the fact that the assertion is known for the pair $\left(\mathbb{A}^{\operatorname{dim}(X)+n}, \mathbb{A}^{\operatorname{dim}(X)} \times D\right)$ by Theorem 2.2. For more details, the reader can consult [KP12, $\S 6]$. 


\section{Remarks on the simplicial version}

Here we explain briefly that the content of this paper is true for the (obvious) simplicial analog of the Binda-Saito cycle complex.

Also, in Remark 2, we briefly mention Miyazaki's result Miy16b that the simplicial version is equivalent to the cubical, at least in the pro setting along the multiples of the divisor $D \subset X$.

The simplicial version could be important because, in addition to the fact that the simplicial terminology is much more preferred in general, it turns out that the Chern classes with modulus from the relative $K$-groups $K_{\geq 0}(X, D)$ to the Nisnevich hypercohomology of the cycle complex factors through the simplicial version [K16, footnote in $\S 2.2$ ].

\subsection{Definition}

Write $\Delta^{p}:=\operatorname{Spec}\left(\mathbb{Z}\left[t_{0}, \ldots, t_{p}\right] /\left(\sum_{i=0}^{p} t_{i}-1\right)\right)$ and $\bar{\Delta}^{p}:=\operatorname{Proj}\left(\mathbb{Z}\left[U, T_{0}, \ldots, T_{p}\right] /(-U+\right.$ $\left.\left.\sum_{i=0}^{p} T_{i}\right)\right)$ so that $\Delta^{p}$ is the non-vanishing locus of $U$ in $\bar{\Delta}^{p}$. Let $\bar{\Delta}_{\infty}^{p}$ be the effective Cartier divisor in $\bar{\Delta}^{p}$ defined by $U=0$. When we work over a base ring, we consider them over the ring.

We now define a simplicial variant of the modulus condition. Let $(X, D)$ be a pair of an algebraic scheme and an effective Cartier divisor.

Definitioin 6.1. A closed subset $V$ of $X \times \Delta^{p}$ is said to satisfy the modulus condition if the following is true: Let $\bar{V}^{N}$ be the normalization of the (reduced) closure $\bar{V}$ of $V$ in $X \times \bar{\Delta}^{p}$. Then the inequality of Cartier divisors on $\bar{V}^{N}$

$$
\left.D\right|_{\bar{V}^{n}} \leq\left.\bar{\Delta}_{\infty}^{p}\right|_{\bar{V}^{n}}
$$

holds, where $\left.(-)\right|_{\bar{V}^{n}}$ denotes the pull-back to $\bar{V}^{N}$.

Pull-back along the structure morphisms $\Delta^{q} \rightarrow \Delta^{p}$ of the cosimplicial scheme $\left\{\Delta^{p}\right\}_{p}$ turns out to preserve the modulus condition: There is no problem with face maps (taking "containment lemma" for granted), and the degeneracy maps are admissible in the sense that if $\bar{\Gamma}$ is the closure of the graph of the morphism in $\bar{\Delta}^{q} \times \bar{\Delta}^{p}$, then the inequality of Cartier divisors on $\bar{\Gamma}^{N}$ :

$$
\left.\bar{\Delta}_{\infty}^{q}\right|_{\bar{\Gamma}^{N}} \geq\left.\bar{\Delta}_{\infty}^{p}\right|_{\bar{\Gamma}^{N}}
$$

holds. This is a sufficient condition for the preservation of the modulus condition, and it can be shown that the admissibility may be checked after replacing the source by a surjective proper scheme over it equipped with the pull-back of the Cartier divisor, both by [KP12, Lem.2.2]. We sketch how to verify the admissibility: By coordinate change it suffices to consider the projection $\mathbb{A}^{n+1}=\operatorname{Spec}\left(k\left[x_{1}, \ldots, x_{n+1}\right]\right) \rightarrow \mathbb{A}^{n}=\operatorname{Spec}\left(k\left[x_{1}, \ldots, x_{n}\right]\right)$ discarding the last factor. The ill-defined locus of the corresponding rational map between compactifications $\mathbb{P}^{n+1}=\operatorname{Proj}\left(k\left[X_{0}, \ldots, X_{n+1}\right]\right) \rightarrow \mathbb{P}^{n}=\operatorname{Proj}\left(k\left[X_{0}, \ldots, X_{n}\right]\right)$ is the closed subscheme $V\left(X_{0}, \ldots, X_{n}\right)$. If we blow it up, the rational map becomes a morphism; the blow-up is covered by $(n+1)$-dimensional affine spaces 
$\operatorname{Spec}\left(k\left[\frac{X_{0}}{X_{j}}, \ldots, \frac{X_{n}}{X_{j}}, \frac{X_{j}}{X_{n+1}}\right]\right)$ and other less important open subsets. On this open subset, the pull-back of $\bar{\Delta}_{\infty}^{n+1}$ amounts to the divisor $\left(\frac{X_{0}}{X_{j}} \cdot \frac{X_{j}}{X_{n+1}}\right)$, while that of $\bar{\Delta}_{\infty}^{n}$ amounts to $\left(\frac{X_{0}}{X_{j}}\right)$. This verifies the admissibility.

Definitioin 6.2. Let $z_{\text {simp }}^{i}(X \mid D, p)$ be the group of codimension $i$ cycles on $X \times \Delta^{p}$ whose support satisfies the modulus condition and the face condition. This gives us a simplicial abelian group $z_{\text {simp }}^{i}(X \mid D, \bullet)$.

\subsection{Moving lemma}

The analogue of Theorem 1.6 holds for the simplicial variant. For this, the only new point is to establish an analogue of Proposition 2.3 (see below for the analogue of the cheaper way as in Remark 1). We state and prove it now.

Let $\mathcal{O}_{K}$ be a discrete valuation ring and $\pi \in \mathcal{O}_{K}$ in the maximal ideal. For a vector $\boldsymbol{v} \in \mathbb{A}^{n}\left(\mathcal{O}_{K}\right)$ and an integer $s \geq 0$, let $\Psi_{\boldsymbol{v}, s}$ be the morphism

$$
\begin{aligned}
\Psi_{\boldsymbol{v}, s}: \mathbb{A}_{\mathcal{O}_{K}}^{n} \times \mathbb{A}^{1} & \rightarrow \mathbb{A}_{\mathcal{O}_{K}}^{n} \\
(\boldsymbol{x}, t) & \mapsto\left(\boldsymbol{x}+t \pi^{s} \boldsymbol{v}\right) .
\end{aligned}
$$

We are interested in the process of pulling back a cycle in $\mathbb{A}_{\mathcal{O}_{K}}^{n} \times \Delta^{p}$ by $\Psi_{\boldsymbol{v}, s} \times$ $\operatorname{id}_{\Delta^{p}}$ and then by one of the usual triangulation maps $\mathbb{A}_{\mathcal{O}_{K}}^{n} \times \Delta^{p+1} \underset{\operatorname{id}_{\mathfrak{A}_{K}^{n}}^{n} \times \rho_{i}}{\cong}$ $\mathbb{A}_{\mathcal{O}_{K}}^{n} \times\left(\mathbb{A}^{1} \times \Delta^{p}\right), i=1, \ldots, p+1$.

Proposition 6.3. Given a closed subset $V$ of $\mathbb{A}_{\mathcal{O}_{K}}^{n} \times \Delta^{p}$ satisfying the modulus condition with respect to the divisor $V(\pi)$, There is an integer $s(V) \geq 0$ such that the pull-back

$$
\left(\operatorname{id}_{\mathbb{A}_{K}^{n}} \times \rho_{i}\right)^{-1}\left(\Psi_{\boldsymbol{v}, s} \times \operatorname{id}_{\Delta^{p}}\right)^{-1}(V)
$$

satisfies the modulus condition in $\mathbb{A}_{\mathcal{O}_{K}}^{n} \times \Delta^{p+1}$ for every $\boldsymbol{v}$ and $s \geq s(V)$.

Proof. By coordinate change, it suffices to consider the following morphism: $\Delta^{p+1} \cong \operatorname{Spec}\left(\mathbb{Z}\left[t_{1}, \ldots, t_{p+1}\right]\right), \Delta^{p} \cong \operatorname{Spec}\left(\mathbb{Z}\left[t_{1}, \ldots, t_{p}\right]\right)$ and the map is given by

$$
\begin{array}{ccc}
\mathbb{A}_{\mathcal{O}_{K}}^{n} \times \Delta^{p+1} & \rightarrow & \mathbb{A}_{\mathcal{O}_{K}}^{n} \times \Delta^{p} \\
\left(\boldsymbol{x}, t_{1}, \ldots, t_{p+1}\right) & \mapsto & \left(\boldsymbol{x}+t_{p+1} \pi^{s} \boldsymbol{v}, t_{1}, \ldots, t_{p}\right)
\end{array}
$$

which we denote by $\Psi$ anew. Denote the compactifications by

$$
\bar{\Delta}^{p+1}:=\operatorname{Proj}\left(\mathbb{Z}\left[U, T_{1}, \ldots, T_{p+1}\right]\right) \text { and } \bar{\Delta}^{p}:=\operatorname{Proj}\left(\mathbb{Z}\left[U, T_{1}, \ldots, T_{p}\right]\right) .
$$

Modulus condition can be checked after blowing up the source, just like the admissibility of a morphism, by KP12, Lem.2.2]. We blow up $\bar{\Delta}^{p+1}$ by the ideal $\left(U, T_{1}, \ldots, T_{p}\right)$ so that the rational map $\bar{\Delta}^{p+1} \rightarrow \bar{\Delta}^{p}$ becomes well-defined.

The scheme $\bar{\Delta}^{p+1}$ is covered by the open subsets where one of $U, T_{1}, \ldots$, $T_{p+1}$ is invertible. Over the subset $\{U \neq 0\}=\Delta^{p+1}$, there is nothing to 
check because one easily sees that the pull-back of $\bar{V}$ does not meet the pullback of $D$ in this region. The blow-up of the other open sets are covered by $(p+1)$-dimensional affine spaces $\tilde{\Delta}_{i j}^{p+1}$ having $\frac{U}{T_{j}}, \frac{T_{1}}{T_{j}}, \ldots, \frac{\widehat{T_{i}}}{T_{j}}, \ldots, \frac{T_{p+1}}{T_{j}}, \frac{T_{j}}{T_{i}}$ as coordinates, where $i=1, \ldots, p+1$ and $j=1, \ldots, p$ with $i \neq j$.

Now we have a morphism induced by $\Psi$ :

$$
\begin{aligned}
\Psi & : \mathbb{A}_{\mathcal{O}_{K}}^{n} \times\left(\tilde{\Delta}_{i j}^{p+1} \backslash\left\{\frac{U}{T_{j}}=0\right\}\right) \\
& \rightarrow \mathbb{A}_{\mathcal{O}_{K}}^{n} \times \operatorname{Spec}\left(\mathbb{Z}\left[\frac{U}{T_{i}}, \frac{T_{1}}{T_{i}}, \ldots, \frac{T_{p}}{T_{i}}\right]\right) \quad\left(\stackrel{\text { open }}{\subset} \mathbb{A}_{\mathcal{O}_{K}}^{n} \times \bar{\Delta}^{p}\right) .
\end{aligned}
$$

whose first component is given by $\boldsymbol{x}+\left(\frac{T_{p+1}}{T_{j}} / \frac{U}{T_{j}}\right) \pi^{s} \boldsymbol{v}$, and the second by the ring homomorphism $\frac{(-)}{T_{i}} \mapsto \frac{(-)}{T_{j}} \cdot \frac{T_{j}}{T_{i}}$ where $(-)=U, T_{1}, \ldots, T_{p}$. Let us denote by $\bar{V}_{i}$ the intersection of $\bar{V}$ and the target. We now have to pull it back, take its closure in $\mathbb{A}_{\mathcal{O}_{K}}^{n} \times \tilde{\Delta}_{i j}^{p+1}$ and verify the inequality of Cartier divisors on its normalization required for the modulus condition.

Let $\left\{f_{\lambda}\left(\boldsymbol{x}, \frac{U}{T_{i}}, \frac{T_{1}}{T_{i}}, \ldots, \frac{T_{p}}{T_{i}}\right)\right\}_{\lambda}$ be finitely many functions defining $\bar{V}_{i}$ in the target. Denote by $\operatorname{deg}_{\boldsymbol{x}}\left(f_{\lambda}\right)$ the total degree in the variables $\boldsymbol{x}$. Then the defining ideal for $\left(\Psi^{-1} \bar{V}_{i}\right)^{-}$in $\mathbb{A}_{\mathcal{O}_{K}}^{n} \times \tilde{\Delta}_{i j}^{p+1}$ contains functions

$$
\varphi_{\lambda}:=\left(\frac{U}{T_{j}}\right)^{\operatorname{deg}_{\boldsymbol{x}}\left(f_{\lambda}\right)} \cdot f_{\lambda}\left(\boldsymbol{x}+\left(\frac{T_{p+1}}{T_{j}} / \frac{U}{T_{j}}\right) \pi^{s} \boldsymbol{v}, \frac{U}{T_{j}} \frac{T_{j}}{T_{i}}, \ldots, \frac{T_{p}}{T_{j}} \frac{T_{j}}{T_{i}}\right) .
$$

Expanding it with respect to the first entry, it has the form

$$
\begin{aligned}
\varphi_{\lambda}= & \left(\frac{U}{T_{j}}\right)^{\operatorname{deg}_{\boldsymbol{x}}\left(f_{\lambda}\right)} \cdot f_{\lambda}\left(\boldsymbol{x}, \frac{U}{T_{j}} \frac{T_{j}}{T_{i}}, \ldots, \frac{T_{p}}{T_{j}} \frac{T_{j}}{T_{i}}\right) \\
& +\pi^{s} g_{\lambda}\left(\boldsymbol{x}, \frac{U}{T_{j}}, \ldots, \frac{\widehat{T_{i}}}{T_{j}}, \ldots, \frac{T_{p+1}}{T_{j}}, \frac{T_{j}}{T_{i}}\right)
\end{aligned}
$$

where $g_{\lambda}$ is a polynomial $\in \mathcal{O}_{K}\left[\boldsymbol{x}, \frac{U}{T_{j}}, \ldots, \frac{\widehat{T_{i}}}{T_{j}}, \ldots, \frac{T_{p+1}}{T_{j}}, \frac{T_{j}}{T_{i}}\right]$.

On the other hand, since $V$ is assumed to satisfy the modulus condition, on $\mathbb{A}_{\mathcal{O}_{K}}^{n} \times \operatorname{Spec}\left(\mathbb{Z}\left[\frac{U}{T_{i}}, \frac{T_{1}}{T_{i}}, \ldots, \frac{T_{p}}{T_{i}}\right]\right)$ we have a relation of the form

$$
E_{i}\left(\frac{U}{T_{i}}, \pi\right)=\sum_{\lambda} b_{\lambda} f_{\lambda}
$$

where $E_{i}(\alpha, \beta) E_{i}\left[\boldsymbol{x}, \frac{U}{T_{i}}, \ldots \frac{T_{p}}{T_{i}}\right](\alpha, \beta) \in\left(\mathcal{O}_{K}\left[\boldsymbol{x}, \frac{U}{T_{i}}, \ldots, \frac{T_{p}}{T_{i}}\right]\right)[\alpha, \beta]$ is a polynomial monic in $\alpha$ and homogeneous in $\alpha, \beta$, and $b_{\lambda} \in \mathcal{O}_{K}\left[\boldsymbol{x}, \frac{U}{T_{i}}, \ldots, \frac{T_{p}}{T_{i}}\right]$. 
We apply the $\mathcal{O}_{K}[\boldsymbol{x}]$-algebra homomorphism $\frac{(-)}{T_{i}} \mapsto \frac{(-)}{T_{j}} \frac{T_{j}}{T_{i}}$ to get

$$
\begin{aligned}
\tilde{E}_{i}\left(\frac{U}{T_{j}} \frac{T_{j}}{T_{i}}, \pi\right) & :=E_{i}\left[\boldsymbol{x}, \frac{U}{T_{j}} \frac{T_{j}}{T_{i}}, \ldots \frac{T_{p}}{T_{j}} \frac{T_{j}}{T_{i}}\right]\left(\frac{U}{T_{j}} \frac{T_{j}}{T_{i}}, \pi\right) \\
& =\sum_{\lambda} b_{\lambda}\left(\boldsymbol{x}, \frac{U}{T_{j}} \frac{T_{j}}{T_{i}}, \ldots, \frac{T_{p}}{T_{j}} \frac{T_{j}}{T_{i}}\right) f_{\lambda}\left(\boldsymbol{x}, \frac{U}{T_{j}} \frac{T_{j}}{T_{i}}, \ldots, \frac{T_{p}}{T_{j}} \frac{T_{j}}{T_{i}}\right)
\end{aligned}
$$

Now we assume $s \geq \operatorname{deg}_{\boldsymbol{x}}\left(f_{\lambda}\right)$ for all $\lambda$, and take $\sum_{\lambda} b_{\lambda}\left(\frac{U}{T_{j}}\right)^{s-\operatorname{deg}_{\boldsymbol{x}}\left(f_{\lambda}\right)} \cdot(-)$ of formulas (8); by formula (9), we get

(Something in the defining ideal for $\left(\Psi^{-1} \bar{V}_{i}\right)^{-}$)

$$
=\left(\frac{U}{T_{j}}\right)^{s} \tilde{E}_{i}\left(\frac{U}{T_{j}} \frac{T_{j}}{T_{i}}, \pi\right)+\pi^{s}\left(\frac{U}{T_{j}}\right)^{s-\max _{\lambda}\left\{\operatorname{deg}_{x}\left(f_{\lambda}\right)\right\}} \tilde{g}
$$

with $\tilde{g} \in \mathcal{O}_{K}\left[\boldsymbol{x}, \frac{U}{T_{j}}, \ldots, \frac{\widehat{T_{i}}}{T_{j}}, \ldots, \frac{T_{p+1}}{T_{j}}, \frac{T_{j}}{T_{i}}\right]$. We multiply both sides by $\left(\frac{T_{j}}{T_{i}}\right)^{s}$; then if $2 s-\max _{\lambda}\left\{\operatorname{deg}_{\boldsymbol{x}}\left(f_{\lambda}\right)\right\} \geq s+\operatorname{deg}\left(E_{i}\right)$ where $\operatorname{deg}\left(E_{i}\right)$ denotes the homogeneous degree in $\alpha, \beta$, the right hand side becomes the form $\tilde{E}_{i}^{\prime}\left(\frac{U}{T_{j}} \frac{T_{j}}{T_{i}}, \pi\right)$ with $\tilde{E}_{i}^{\prime}(\alpha, \beta)$ yet another homogeneous polynomial of degree $s+\operatorname{deg}\left(\tilde{E}_{i}\right)$.

Therefore if we let $s(V)$ the maximum of numbers $\operatorname{deg}\left(E_{i}\right)+\operatorname{deg}_{\boldsymbol{x}}\left(f_{\lambda}\right)$ (the index set for $\lambda$ varies with $i$ ), our assertion holds.

Remark 2. As is the case with the cubical version, the moving lemma for the simplicial version can be proved using $\Psi_{\boldsymbol{v}, 1}$ and push-forward by high enough powers $\mathbb{A}^{1} \rightarrow \mathbb{A}^{1}$. For this, it is convenient to use a kind of sup-modulus condition on $\mathbb{A}_{\mathcal{O}_{K}}^{n} \times \mathbb{A}^{1} \times \Delta^{p}$ where a closed set $V$ is said to satisfy the modulus condition if locally on the normalization $\bar{V}^{N}$ of the closure of $V$ in $\mathbb{A}_{\mathcal{O}_{K}}^{n} \times \mathbb{P}^{1} \times \bar{\Delta}^{p}$, either (where $D$ is the divisor defined by the function $\pi$ )

$$
\left.D\right|_{\bar{V}^{N}} \leq\left. F_{1}\right|_{\bar{V}^{N}} \quad \text { or }\left.\quad D\right|_{\bar{V}^{N}} \leq\left.\bar{\Delta}_{\infty}^{p}\right|_{\bar{V}^{N}}
$$

holds; then it can be checked that the modulus condition is preserved by pullback along triangulation maps $\Delta^{p+1} \cong \mathbb{A}^{1} \times \Delta^{p}$.

Let me mention the issue of the equivalence of cubical and simplicial version. Miyazaki Miy16b has proved that the cubical and simplicial higher Chow groups with modulus are isomorphic if we go pro over the multiples of $D \subset X$. In the proof, he considers a double complex $z^{i}(X ; p, q)$ of cycles in $X \times \Delta^{p} \times \square^{q}$, and the two associated spectral sequences, just as in Blo98.

One of the two spectral sequences degenerates thanks to the cube invariance of the cubical version ( Miy16a, extended from the minus cube to pairs $\left.\left(\bar{\Delta}^{p},-\bar{\Delta}_{\infty}^{p}\right)\right)$. He observes that the simplicial version satisfies the cube invariance at least in pro; this is because the triangulation maps $\Delta^{p+1} \cong \mathbb{A}^{1} \times \Delta^{p}$ are not admissible but they are, up to doubling the divisor on the source. This implies that the other spectral sequence degenerates at least in pro, which enables him to establish a parallel of the argument in Blo98. 


\section{References}

[AGV71] Michael Artin, Alexander Grothendieck, and Jean-Louis Verdier. Theorie de Topos et Cohomologie Etale des Schemas I, II, III, volume 269, 270, 305 of Lecture Notes in Mathematics. Springer, 1971.

[BE03] Spencer Bloch and Hélène Esnault. An additive version of higher Chow groups. Annales Scientifiques de l'École Normale Suprieure, 36(3):463-477, 2003.

[Blo86] Spencer Bloch. Algebraic cycles and higher $K$-theory. Advances in Mathematics, 61(3):267-304, 1986.

[Blo98] Spencer Bloch. Some notes on elementary properties of higher chow groups, including functoriality properties and cubical chow groups. Notes available on his web page, (14 pages), presumably around 1998.

[BS17] Federico Binda and Shuji Saito. Relative cycles with moduli and regulator maps. Journal of the Institute of Mathematics of Jussieu, 16(1):1-61, 2017.

[Cho56] Wei-Liang Chow. On equivalence classes of cycles in an algebraic variety. Annals of Mathematics, 64(3):450-479, 1956.

[DG67] Jean Dieudonné and Alexander Grothendieck. Éléments de géométrie algébrique. Inst. Hautes Études Sci. Publ. Math., 4, 8, 11, 17, 20, 24, 28, 32, 1961-1967.

[IK16] Ryomei Iwasa and Wataru Kai. Chern classes with modulus. ArXiv e-prints, arXiv:1611.07882, November 2016.

[Iwa17] Ryomei Iwasa. Some results on relative $K_{0}$, and relative cycle class map with modulus. arXiv, 1706.08935, 2017.

[KP12] Amalendu Krishna and Jinhyun Park. Moving lemma for additive higher Chow groups. Algebra \&3 Number Theory, 6(2):293-326, 2012.

[KP15] Amalendu Krishna and Jinhyun Park. Algebraic cycles and crystalline cohomology. ArXiv e-prints, arXiv:1504.08181, April 2015.

[KP17] Amalendu Krishna and Jinhyun Park. A module structure and a vanishing theorem for cycles with modulus. Mathematical Research Letters, 24(4):1147-1176, 2017.

[KS16] Moritz Kerz and Shuji Saito. Chow group of 0-cycles with modulus and higher-dimensional class field theory. Duke Math. J., 165(15):2811-2897, 102016.

[Lev98] Marc Levine. Mixed Motives, volume 57 of Mathematical surveys and monographs. American Mathematical Society, 1998. 
[Lev06] Marc Levine. Chow's moving lemma and the homotopy coniveau tower. K-Theory, 37(1-2):129-209, 2006.

[Lev08] Marc Levine. The homotopy coniveau tower. Journal of Topology, 1(1):217-267, 2008.

[Miy16a] Hiroyasu Miyazaki. Cube invariance of higher Chow groups with modulus. ArXiv e-prints, arXiv:1604.06155v3, April 2016.

[Miy16b] Hiroyasu Miyazaki. Private communication. March 2016.

[Par09] Jinhyun Park. Regulators on additive higher Chow groups. American Journal of Mathematics, 131(1):257-276, 2009.

[Rob71] Joel Roberts. Generic projections of algebraic varieties. American Journal of Mathematics, 93:191-214, 1971.

[Ros52] Maxwell Rosenlicht. Equivalence relations on algebraic curves. Annals of Mathematics, 56(1):169-191, 1952.

[RS15] Kay Rülling and Shuji Saito. Higher Chow groups with modulus and relative Milnor K-theory. arXiv, 1504.02669v2, 2015.

[Rül07] Kay Rülling. The generalized de Rham-Witt complex over a field is a complex of zero-cycles. J. Algebraic Geom., (16):109-169, 2007.

[Rus13] Henrik Russell. Albanese varieties with modulus over a perfect field. Algebra \&3 Number Theory, 7(4):853-892, 2013.

[Ser75] Jean-Pierre Serre. Algèbre Locale, Multiplicités, volume 11 of Lecture Notes in Mathematics. Springer-Verlag Berlin Heidelberg, 3rd edition, 1975 .

[SS18] Johannes Schmidt and Florian Strunk. Stable $\mathbb{A}^{1}$-connectivity over dedekind schemes. Annals of K-Theory, 3(2):331-367, 2018. 